\title{
Chemotherapy-Induced Peripheral Neuropathy in Survivors of Adult Cancers: ASCO Guideline Update
}

- Charles L. Loprinzi, MD²; Christina Lacchetti, MHSc ${ }^{2}$; Jonathan Bleeker, MD³ ${ }^{3}$ Guido Cavaletti, MD, PhD ${ }^{4}$; Cynthia Chauhan, MSW5; Daniel L. Hertz, PharmD, PhD ${ }^{6}$; Mark R. Kelley, PhD; Antoinette Lavino, BS Pharm, RPh ${ }^{8}$; Maryam B. Lustberg, MD ${ }^{9}$;

Judith A. Paice, PhD, RN ${ }^{10}$; Bryan P. Schneider, MD ${ }^{11}$; Ellen M. Lavoie Smith, RN, PhD ${ }^{6}$; Mary Lou Smith, JD, MBA ${ }^{12}$; Thomas J. Smith, MD ${ }^{13}$; Nina Wagner Johnston, MD ${ }^{13}$; and Dawn L. Hershman, MD ${ }^{14}$

PURPOSE To update the ASCO guideline on the recommended prevention and treatment approaches in the management of chemotherapy-induced peripheral neuropathy (CIPN) in adult cancer survivors.

METHODS An Expert Panel conducted targeted systematic literature reviews to identify new studies.

RESULTS The search strategy identified 257 new references, which led to a full-text review of 87 manuscripts. A total of 3 systematic reviews, 2 with meta-analyses, and 28 primary trials for prevention of CIPN in addition to 14 primary trials related to treatment of established CIPN, are included in this update.

RECOMMENDATIONS The identified data reconfirmed that no agents are recommended for the prevention of CIPN. The use of acetyl-L-carnitine for the prevention of CIPN in patients with cancer should be discouraged. Furthermore, clinicians should assess the appropriateness of dose delaying, dose reduction, substitutions, or stopping chemotherapy in patients who develop intolerable neuropathy and/or functional impairment. Duloxetine is the only agent that has appropriate evidence to support its use for patients with established painful CIPN. Nonetheless, the amount of benefit from duloxetine is limited.

Additional information is available at www.asco.org/survivorship-guidelines.

J Clin Oncol 38:3325 3348. $\odot 2020$ by American Society of Clinical Oncology

ASSOCIATED

CONTENT

Appendix

Data Supplement

Author affiliations

and support

information (if

applicable) appear

at the end of this

article.

Accepted on May 15, 2020 and published at ascopubs.org/journal/ jco on July 14,2020 : DOI https://doi.org/10. 1200/JC0.20.01399

Clinical Practice Guidelines

Committee approval: May 5, 2020

Reprint Requests: 2318 Mill Rd, Suite 800, Alexandria, VA 22314; guidelines@ asco.org

\section{INTRODUCTION}

Chemotherapy-induced neuropathy is a serious clinical problem caused by a substantial number of cytotoxic drugs, including taxanes, platinums, vinca alkaloids, epothilones, eribulin, and bortezomib; these drugs cause different pathologic insults to neurons. Although there are differences and similarities between the neuropathies caused by these agents, historically, they have not been well defined. Inconsistent measurement methods have often been used to characterize the variations in neuropathy caused by different chemotherapy drugs. However, the same validated neuropathy measurement tools have been recently used in several clinical studies. The data arising from these studies allow for a more detailed comparison of neuropathy clinical manifestations caused by two of the most prominent neurotoxic chemotherapy agents, paclitaxel and oxaliplatin. ${ }^{1-4}$

Both oxaliplatin and paclitaxel cause acute neuropathy. Oxaliplatin-induced acute neuropathy is characterized by cold sensitivity, throat discomfort, discomfort swallowing cold liquids, and muscle cramps. Although some of these symptoms can occur within the time of drug infusion, their severity usually peaks 2 to 3 days after each dose of oxaliplatin. With subsequent treatment cycles, symptom severities double in magnitude over that seen for the first treatment cycle. Oxaliplatininduced acute neuropathy does not return to baseline between cycles when oxaliplatin is administered once every 2 weeks. There is no good information to delineate how long acute symptoms last after the last dose of oxaliplatin.

Paclitaxel also frequently causes a pain syndrome that occurs in the days following each dose. These symptoms, in the past, had been labeled as being arthralgias or myalgias. However, newer data support that they are a manifestation of an acute neuropathy. ${ }^{2,5}$ These acute neuropathy symptoms from paclitaxel present with a similar time pattern as oxaliplatin acute neuropathy symptoms, peaking approximately 2 to 3 days after each dose of paclitaxel. The symptom complex, however, is different than that seen with oxaliplatin, in that it is primarily a pain, classically occurring in a truncal/hip distribution. In comparison 


\section{THE BOTTOM LINE}

Prevention and Management of Chemotherapy-Induced Peripheral Neuropathy in Survivors of Adult Cancers: ASCO Guideline Update

\section{Guideline Question}

What are the recommended prevention and treatment approaches in the management of chemotherapy-induced neuropathies in adult cancer survivors?

\section{Target Population}

Adult cancer survivors with, or at risk for developing, chemotherapy-induced neuropathies

\section{Target Audience}

Health care practitioners who provide care to cancer survivors; patients and their caregivers

\section{Methods}

An Expert Panel was convened to update clinical practice guideline recommendations based on a systematic review of the medical literature.

\section{Updated Recommendations}

The following recommendations are evidence based, informed by randomized trials, and guided by clinical experience. The recommendations were developed by a multidisciplinary group of experts.

\section{Prevention of chemotherapy-induced peripheral neuropathy.}

1.1 Clinicians should assess the risks and benefits of agents known to cause CIPN among patients with underlying neuropathy and with conditions that predispose to neuropathy such as diabetes and/or a family or personal history of hereditary neuropathy (Type of recommendation: Informal consensus, benefits outweigh harms; Evidence quality: low; Strength of recommendation: moderate).

1.2 Clinicians should not offer, and should discourage use of, acetyl---carnitine for the prevention of CIPN in patients with cancer (Type of recommendation: evidence based, harms outweigh benefits; Evidence quality: high; Strength of recommendation: strong).

1.3 Outside the context of a clinical trial, no recommendations can be made on the use of the following interventions for the prevention of CIPN:

- Acupuncture

- Cryotherapy

- Compression therapy

- Exercise therapy

- Ganglioside-monosialic acid (GM-1)

(Type of recommendation: no recommendation; Evidence quality: low; Strength of recommendation: not applicable).

Note: While preliminary evidence suggests a potential for benefit from these interventions, larger sample-sized definitive studies are needed to confirm efficacy and clarify risks.

1.4 Clinicians should not offer the following agents for the prevention of CIPN to patients with cancer undergoing treatment with neurotoxic agents:

- All-trans retinoic acid

- Amifostine

- Amitriptyline

- Calcium magnesium

- Calmangafodipir

- Cannabinoids

- Carbamazepine

- L-carnosine

- Diethyldithiocarbamate (DDTC)

- Gabapentin/pregabalin

- Glutamate

- Glutathione (GSH) for patients receiving paclitaxel/carboplatin chemotherapy

- Goshajinkigan (GJG)

- Metformin 


\section{THE BOTTOM LINE (CONTINUED)}

- Minocycline

- $N$-acetylcysteine

- Nimodipine

- Omega-3 fatty acids

- Org 2766

- Oxcarbazepine

- Recombinant human leukemia inhibitory factor

- Venlafaxine

- Vitamin B

- Vitamin E

(Type of recommendation: evidence based, no benefits; Evidence quality: intermediate; Strength of recommendation: moderate).

Treatment of chemotherapy-induced peripheral neuropathy that develops while patients are receiving neurotoxic chemotherapy.

2.1 Clinicians should assess, and discuss with patients, the appropriateness of dose delaying, dose reduction, or stopping chemotherapy (or substituting with agents that do not cause CIPN) in patients who develop intolerable neuropathy and/or functional nerve impairment (Type of recommendation: informal consensus, benefits outweigh harms; Evidence quality: low; Strength of recommendation: moderate).

\section{Treatment of chemotherapy-induced peripheral neuropathy for patients who have completed neurotoxic chemotherapy.}

3.1 For patients with cancer experiencing painful CIPN, clinicians may offer duloxetine (Type of recommendation: evidence based, benefits equal harms; Evidence quality: intermediate; Strength of recommendation: moderate).

3.2 Outside the context of a clinical trial, no recommendations can be made on the use of the following interventions for the treatment of CIPN:

- Exercise therapy

- Acupuncture

- Scrambler therapy

- Gabapentin/pregabalin

- Topical gel treatment containing baclofen, amitriptyline $\mathrm{HCL}$, plus/minus ketamine

- Tricyclic antidepressants

- Oral cannabinoids

(Type of recommendation: no recommendation; Evidence quality: low; Strength of recommendation: not applicable).

Note: While recent preliminary evidence suggests a potential for benefit from exercise, acupuncture, and scrambler therapy, larger sample-sized definitive studies are needed to confirm efficacy and clarify risks.

\section{Additional Resources}

More information, including a supplement with additional evidence tables, slide sets, and clinical tools and resources, is available at www.asco.org/survivorship-guidelines. The Methodology Manual (available at www.asco.org/guidelinemethodology) provides additional information about the methods used to develop this guideline. Patient information is available at www.cancer.net

ASCO believes that cancer clinical trials are vital to inform medical decisions and improve cancer care, and that all patients should have the opportunity to participate.

with oxaliplatin-related symptoms, these symptoms tend to resolve more between doses of paclitaxel, and the symptoms are not worsened, on average, in subsequent cycles.

The chronic neuropathies related to these 2 drugs share several similarities. The neuropathy associated with each drug is primarily sensory, as opposed to motor or autonomic. The most common descriptors of this sensory neuropathy are numbness, tingling, and pain. Numbness and tingling appear earlier and are generally more prominent problems than pain. A stocking-glove distribution of symptoms typically begins distally in the fingers and toes and can progress proximally as the condition worsens.

When comparing chronic neuropathy distribution patterns between the 2 drugs, paclitaxel-induced chronic neuropathy symptoms are more prominent in the lower extremities than upper extremities during treatment. In contrast, 
oxaliplatin-induced symptoms experienced during treatment are more severe in the upper extremities than in the lower extremities.

After completion of chemotherapy treatments, paclitaxel neuropathy, on average, improves over the ensuing several months. In contrast, oxaliplatin-induced neuropathy, on average, worsens for 2-3 months after cessation of therapy (labeled as coasting phenomenon); after approximately 3 months, neuropathy tends to improve. ${ }^{3}$ Neuropathy in the hands improves faster than in the feet, so that, months after completion of oxaliplatin, neuropathy is worse in the feet than in the hands. Although neuropathy caused by both drugs tends to improve over time, neuropathy can remain as a substantial debilitating problem in a subset of patients for years. ${ }^{6,7}$

The diagnosis of the more chronic chemotherapy-induced peripheral neuropathy can generally be made by clinical history. If a patient receiving neurotoxic chemotherapy develops new or worsening numbness, tingling, and/or pain in their hands and/or feet, and there is no other good reason for them to have developed these symptoms, then the diagnosis is made. Neurologic physical examination can be abnormal in a patient with chemotherapy-induced peripheral neuropathy. Neurologic tests, such as electromyography (EMG), can be used but are not usually necessary. There are data supporting that nerve conduction studies in asymptomatic patients who are receiving neurotoxic chemotherapy can predict the development or worsening of chemotherapy-induced peripheral neuropathy (CIPN). ${ }^{8-10}$ These tests, however, are not routinely used.

Chemotherapy-induced peripheral neuropathy can markedly affect the quality of life (QOL) of patients. In addition, it may be detrimental to their cancer outcomes, as it may limit the amount of chemotherapy that clinicians can give.

The purpose of this guideline update is to systematically review new evidence reported in the literature since the original guideline was published, compare outcomes among trials, and provide updated guidance on the effectiveness of prevention and treatment options for CIPN in adults with a history of cancer.

\section{GUIDELINE QUESTIONS}

This clinical practice guideline addresses 2 overarching clinical questions: What are the recommended (1) prevention and (2) treatment approaches in the management of chemotherapy-induced neuropathies in adult cancer survivors?

\section{METHODS}

\section{Guideline Update Development Process}

This systematic review-based guideline was developed by a multidisciplinary expert panel, which included a patient representative and an ASCO guidelines staff member with health research methodology expertise (Appendix Table A1, online only). The Expert Panel met via webinar and corresponded through e-mail. On the basis of the consideration of the evidence, the authors were asked to contribute to the development of the guideline, provide critical review, and finalize the guideline recommendations. The guideline recommendations were made available for an open comment period of 2 weeks, allowing the public to review and comment on the recommendations after submitting a confidentiality agreement. The full guideline was shared with 2 external reviewers. Comments were taken into consideration while finalizing the recommendations. Members of the Expert Panel were responsible for reviewing and approving the penultimate version of the guideline, which was then circulated for external review and submitted to Journal of Clinical Oncology for editorial review and consideration for publication. All ASCO guidelines are ultimately reviewed and approved by the Expert Panel and the ASCO Clinical Practice Guidelines Committee before publication. All funding for the administration of the project was provided by ASCO.

The recommendations were developed using a systematic review and informed by expert clinical experience. PubMed was searched for randomized controlled trials (RCTs) and meta-analyses published between January 1, 2013, and August 28, 2019. An updated search was conducted in February 2020. Search terms are provided in the Data Supplement. Randomized trial articles were selected for inclusion in the systematic review of the evidence if they (1) focused on chemotherapy-induced neuropathy, (2) included cancer survivors, and (3) considered neuropathy as an important outcome of the study. Articles were excluded from the systematic review if they (1) were phase I studies, other noncomparative studies, case reports, editorial letters, or newspaper articles; (2) only involved individuals $<18$ years of age; (3) were published in a language other than English; (4) included < 10 participants; or (5) focused on radiation therapy-related neuropathy or stem-cell transplantation-related neuropathy.

The updated search was guided by the "signals"11 approach that is designed to identify only new, potentially practice-changing data-signals - that might translate into revised practice recommendations. The approach relies on targeted routine literature searching and the expertise of ASCO Expert Panel members to help identify potential signals. Before publication, a review of guideline implementability was also conducted. Ratings for the type and strength of the recommendation and the quality of evidence are provided with each recommendation, using standardized criteria that are applied to all ASCO guidelines. The Methodology Manual (available at www.asco.org/ guideline-methodology) provides additional information about the methods used to develop this guideline update.

The ASCO Expert Panel and guidelines staff will continue to work with co-chairs in the future to keep abreast of the need 
for any substantive updates to the guideline. On the basis of formal review of the emerging literature, ASCO will determine the need to update.

\section{Guideline Disclaimer}

The Clinical Practice Guidelines and other guidance published herein are provided by the American Society of Clinical Oncology, Inc. (ASCO) to assist providers in clinical decision making. The information herein should not be relied upon as being complete or accurate, nor should it be considered as inclusive of all proper treatments or methods of care or as a statement of the standard of care. With the rapid development of scientific knowledge, new evidence may emerge between the time information is developed and when it is published or read. The information is not continually updated and may not reflect the most recent evidence. The information addresses only the topics specifically identified therein and is not applicable to other interventions, diseases, or stages of diseases. This information does not mandate any particular course of medical care. Further, the information is not intended to substitute for the independent professional judgment of the treating provider, as the information does not account for individual variation among patients. Recommendations reflect high, moderate, or low confidence that the recommendation reflects the net effect of a given course of action. The use of words like "must," "must not," "should," and "should not" indicates that a course of action is recommended or not recommended for either most or many patients, but there is latitude for the treating physician to select other courses of action in individual cases. In all cases, the selected course of action should be considered by the treating provider in the context of treating the individual patient. Use of the information is voluntary. ASCO provides this information on an "as is" basis and makes no warranty, express or implied, regarding the information. ASCO specifically disclaims any warranties of merchantability or fitness for a particular use or purpose. ASCO assumes no responsibility for any injury or damage to persons or property arising out of or related to any use of this information, or for any errors or omissions.

\section{Guideline and Conflicts of Interest}

The Expert Panel was assembled in accordance with ASCO's Conflict of Interest Policy Implementation for Clinical Practice Guidelines ("Policy," found at http:// www.asco.org/rwc). All members of the Expert Panel completed ASCO's disclosure form, which requires disclosure of financial and other interests, including relationships with commercial entities that are reasonably likely to experience direct regulatory or commercial impact as a result of promulgation of the guideline. Categories for disclosure include employment; leadership; stock or other ownership; honoraria, consulting or advisory role; speaker's bureau; research funding; patents, royalties, other intellectual property; expert testimony; travel, accommodations, expenses; and other relationships. In accordance with the Policy, the majority of the members of the Expert Panel did not disclose any relationships constituting a conflict under the Policy.

\section{RESULTS}

The review of prevention and treatment of CIPN identified a total of 31 prevention and 14 treatment publications that met eligibility criteria and form the evidentiary basis for the guideline updated recommendations. Characteristics and key results of these publications, by clinical question, are provided in Tables 1 and 2. Studies that were particularly pertinent to the development of the recommendations are discussed in the Literature Review Update and Analysis sections.

\section{Study Quality Assessment}

Study quality was formally assessed for the 45 intervention studies identified. Systematic reviews and meta-analyses were assessed for quality using the AMSTAR tool. ${ }^{12}$ Design elements such as blinding, allocation concealment, sufficient sample size, intention to treat, and funding sources were assessed for RCTs. AMSTAR scores ranged from 8 to 9 out of a possible 11 points. Overall, the included systematic reviews were conducted in a rigorous fashion; however, many of the primary studies included in these reviews suffered from flaws in study design. Additional RCTs identified and included in this guideline ranged from low to high overall risk of bias. Many of these trials also had flaws in the study design, mainly around blinding; had small sample sizes and/or high attrition rates; and lacked statistical power, thus lowering the confidence in the findings. The included studies were also heterogeneous with respect to patient populations, sample size, methodological quality, treatment duration, and outcome measures. The primary outcomes varied across studies and, in the majority of cases, were not directly comparable because of different outcomes, measurements, and instruments used at different time points. This diversity precluded a quantitative analysis and, as such, only a qualitative review was performed. Refer to the Data Supplement for quality rating scores and the Methodology Manual (http://www.asco.org/ guideline-methodology) for definitions of ratings for overall potential risk of bias.

\section{UPDATED RECOMMENDATIONS}

\section{CLINICAL QUESTION}

What are the recommended prevention and treatment approaches in the management of chemotherapy-induced neuropathies in adult cancer survivors?

\section{Prevention of Chemotherapy-Induced Peripheral Neuropathy}

1.1 Clinicians should assess the risks and benefits of agents known to cause CIPN among patients with underlying neuropathy and with conditions that 


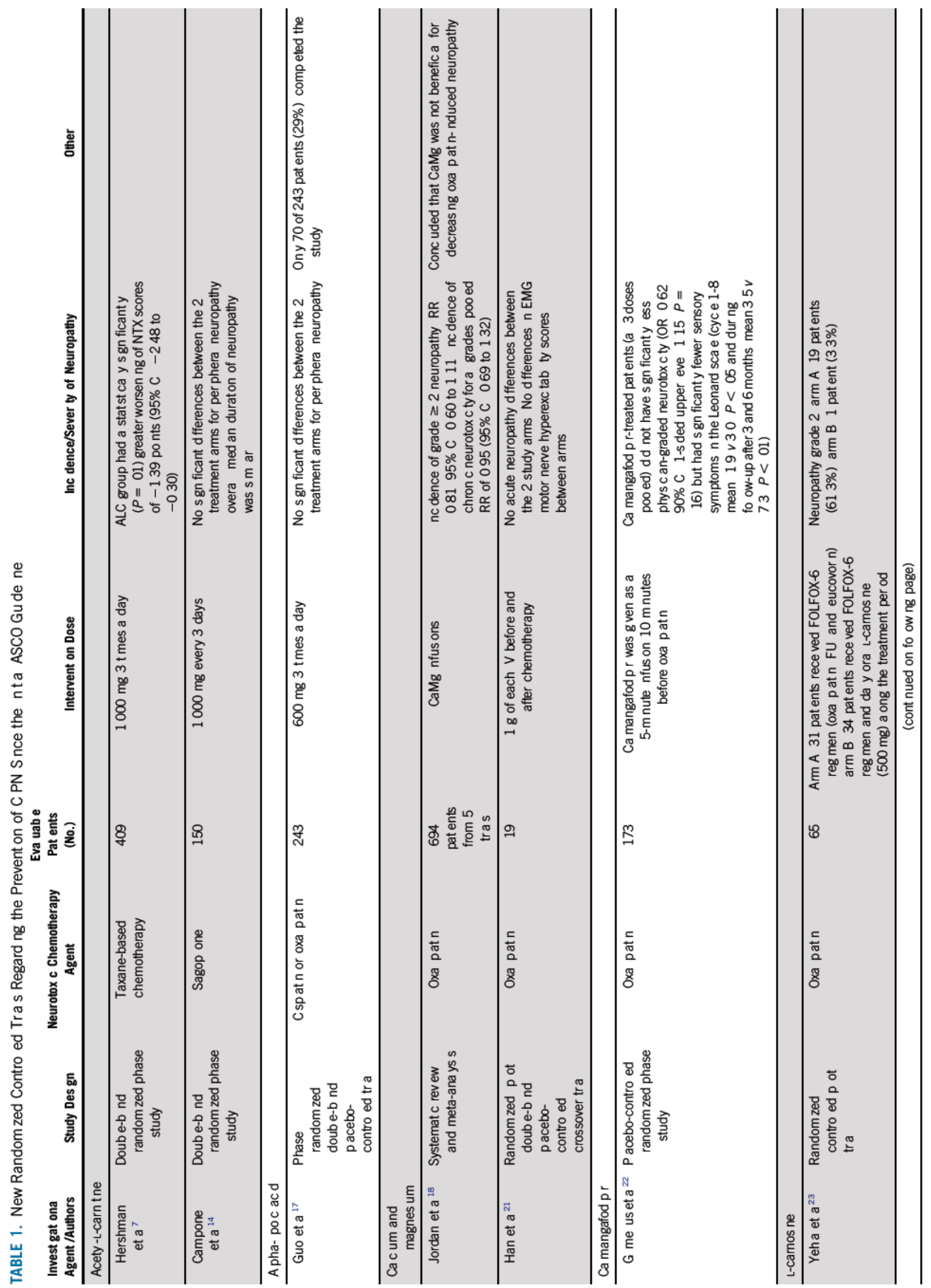




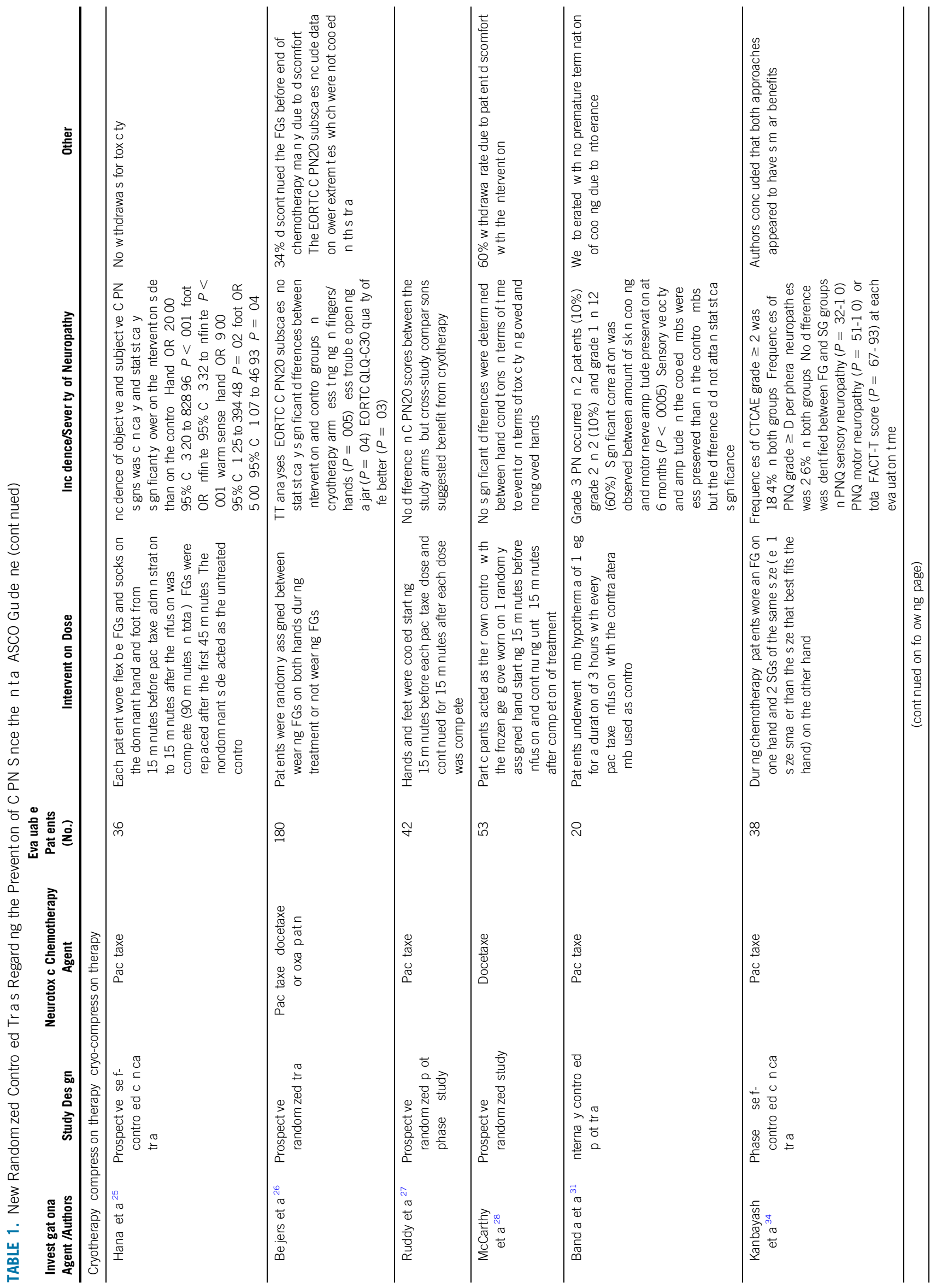




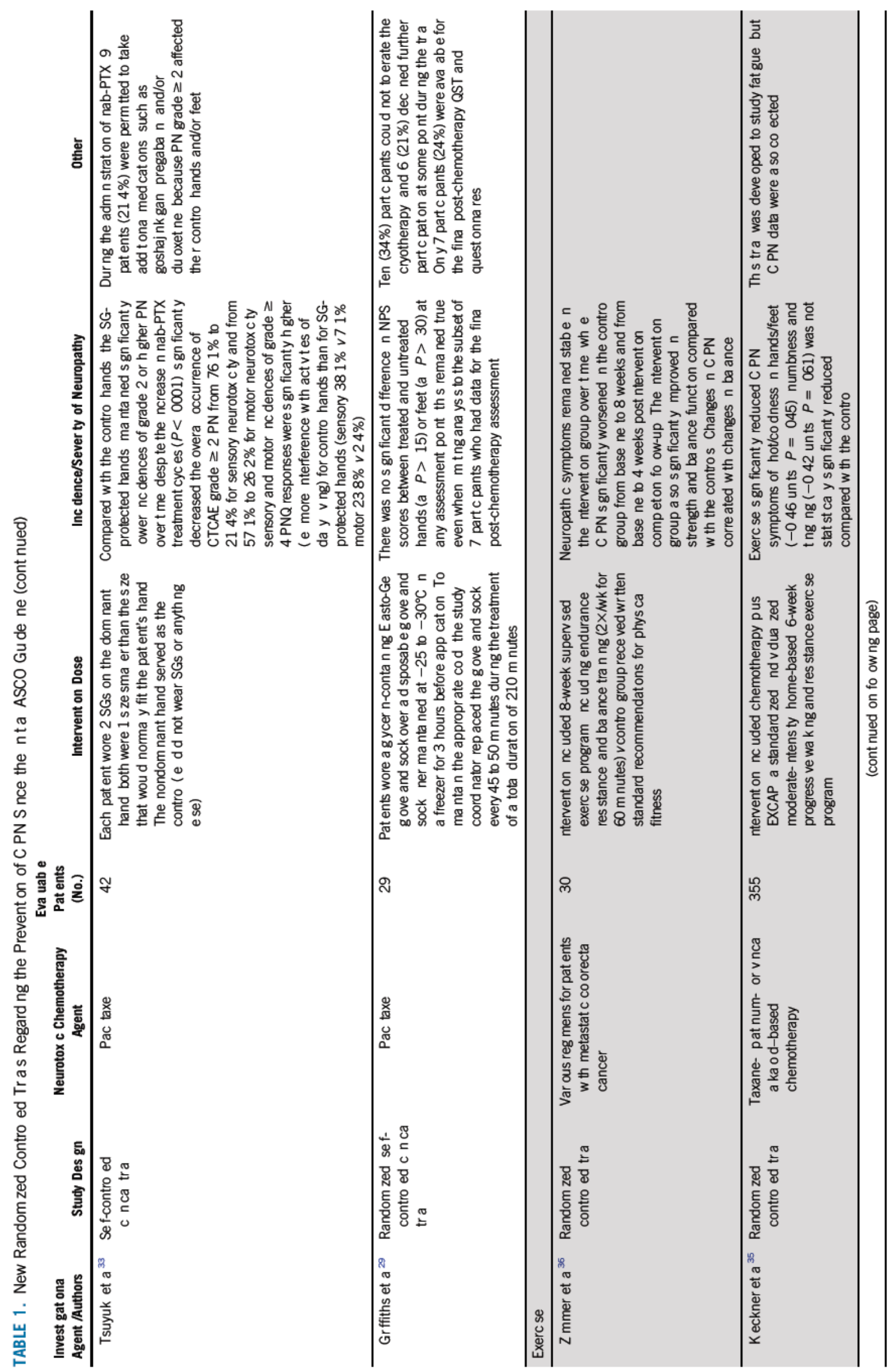




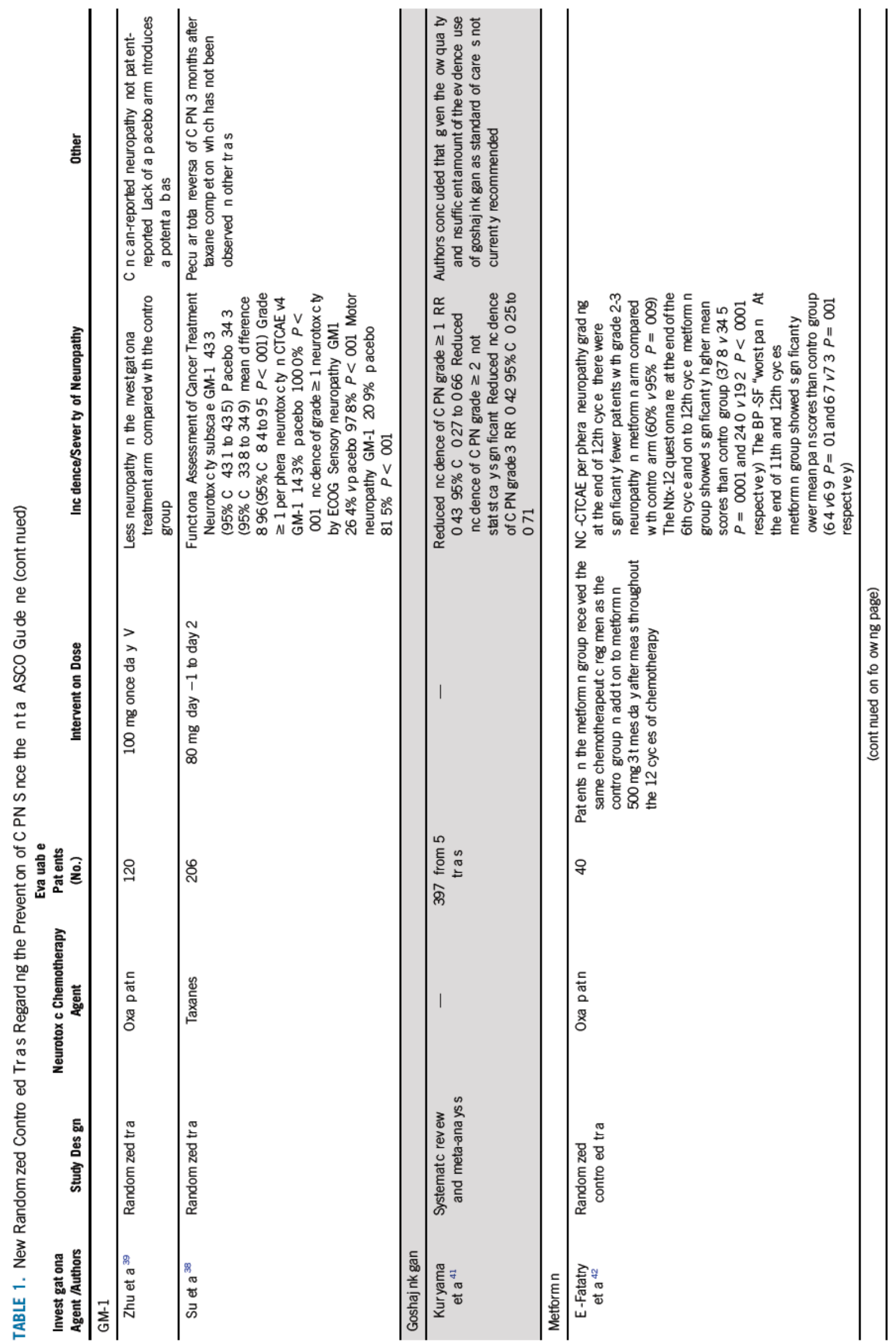




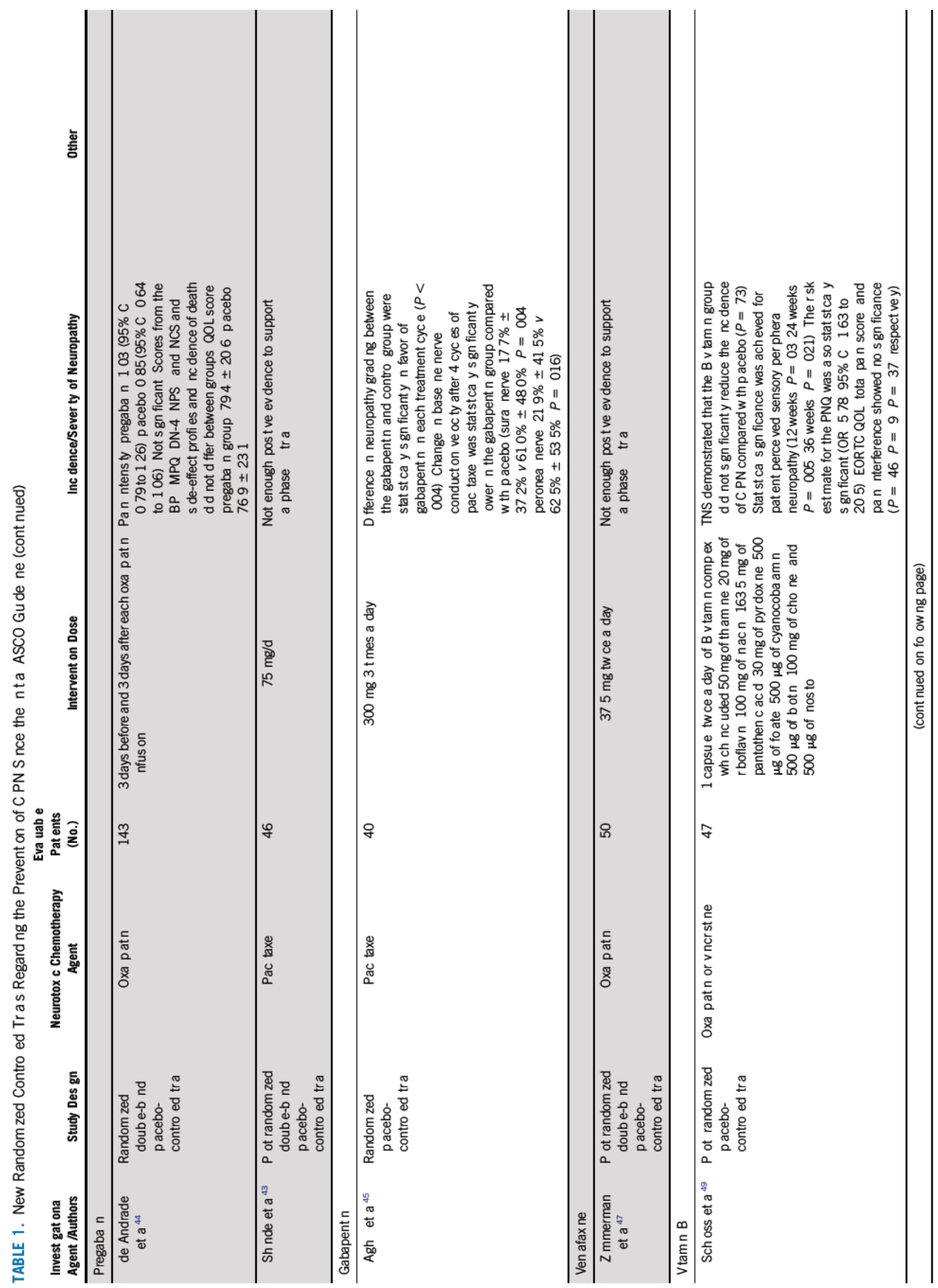




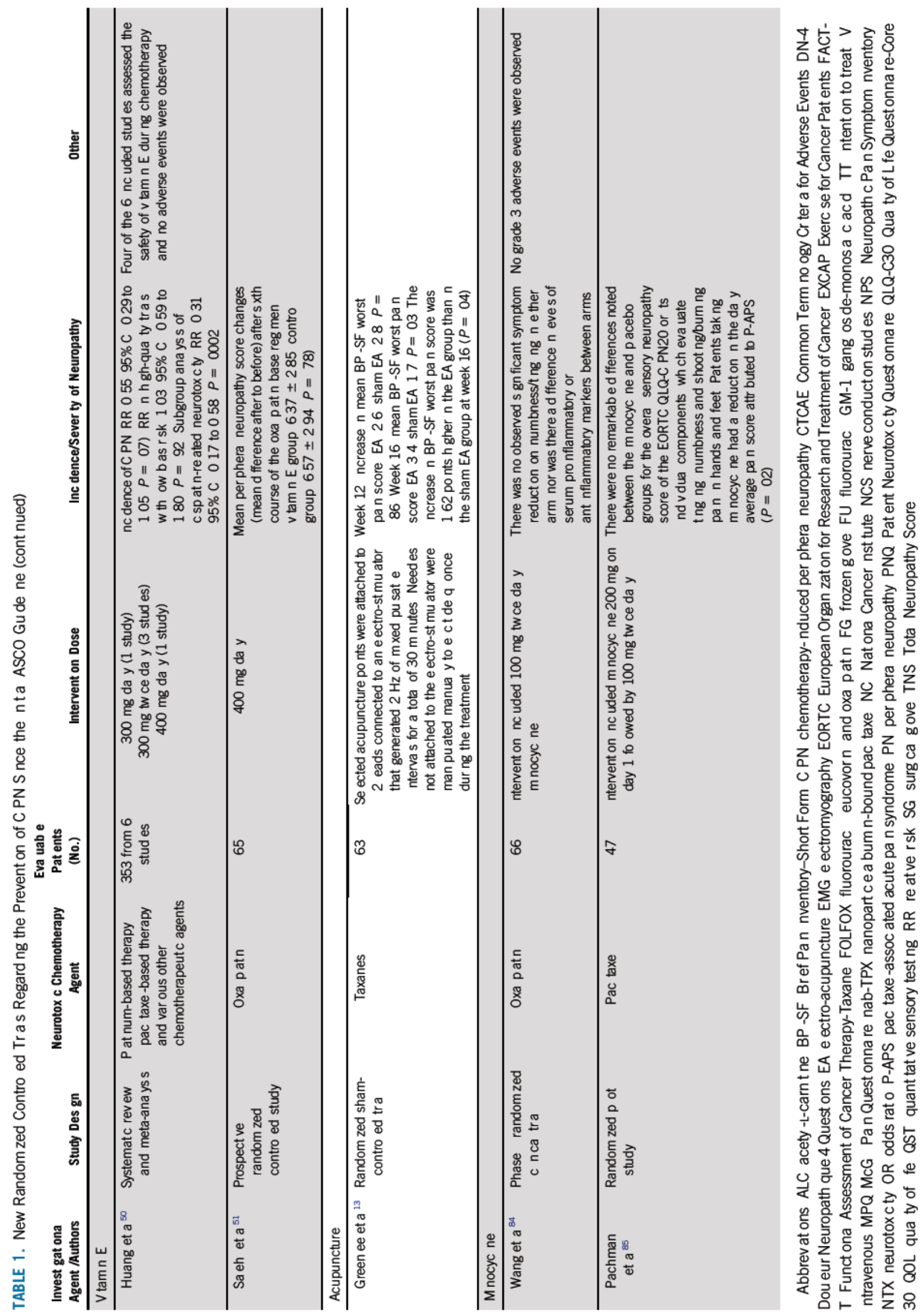


predispose to neuropathy such as diabetes and/or a family or personal history of hereditary peripheral neuropathy (Type of recommendation: informal consensus, benefits outweigh harms; Evidence quality: low; Strength of recommendation: moderate).

1.2 Clinicians should not offer, and should discourage use of, acetyl-L-carnitine for the prevention of CIPN in patients with cancer (Type of recommendation: evidence based, harms outweigh benefits; Evidence quality: high; Strength of recommendation: strong).

1.3 Outside the context of a clinical trial, no recommendations can be made on the use of the following interventions for the prevention of CIPN:

- Acupuncture

- Cryotherapy

- Compression therapy

- Exercise therapy

- Ganglioside-monosialic acid (GM-1)

(Type of recommendation: no recommendation; Evidence quality: low; Strength of recommendation: not applicable).

Note: While preliminary evidence suggests a potential for benefit from these interventions, larger sample-sized definitive studies are needed to confirm efficacy and clarify risks.

1.4 Clinicians should not offer the following agents for the prevention of CIPN to patients with cancer undergoing treatment with neurotoxic agents:

- All-trans retinoic acid

- Amifostine

- Amitriptyline

- Calcium magnesium

- Calmangafodipir

- Cannabinoids

- Carbamazepine

- L-carnosine

- Diethyldithiocarbamate (DDTC)

- Gabapentin/pregabalin

- Glutamate

- Glutathione (GSH) for patients receiving paclitaxel/ carboplatin chemotherapy

- Goshajinkigan (GJG)

- Metformin

- Minocycline

- $N$-acetylcysteine

- Nimodipine

- Omega-3 fatty acids

- Org 2766

- Oxcarbazepine

- Recombinant human leukemia inhibitory factor

- Venlafaxine

- Vitamin B

- Vitamin E

(Type of recommendation: evidence based, no benefits; Evidence quality: intermediate; Strength of recommendation: moderate).

\section{Literature Review Update and Analysis on Prevention}

Acupuncture. One small randomized, sham-controlled trial of weekly electro-acupuncture for the prevention of taxaneinduced peripheral neuropathy in 63 patients did not show any differences in neuropathy between groups. ${ }^{13}$ In this trial, the electro-acupuncture arm actually had a slower recovery of neuropathy than was seen in the sham group, after chemotherapy was stopped.

Acetyl-L-carnitine. Two trials evaluating acetyl-L-carnitine were identified. Campone et $\mathrm{a}^{14}$ reported data on the use of acetyl-L-carnitine for preventing sagopilone-induced neuropathy in 150 patients randomly assigned to receive acetyl-L-carnitine or placebo. There were no significant differences between the 2 treatment arms for peripheral neuropathy overall, and the median duration of neuropathy was similar. ${ }^{14}$ These data are consistent with older data from a previously reported trial in patients receiving paclitaxel, where neuropathy was actually worse in the patients who received acetyl-L-carnitine. ${ }^{15,16}$ In a recent long-term follow-up analysis ${ }^{7}$ of that trial, 24 weeks of acetyl-L-carnitine therapy resulted in statistically significantly worse CIPN ( $P \quad .01$ ) over 2 years, as measured by the Functional Assessment of Cancer Therapy-Neurotoxicity (FACT-Ntx) Questionnaire.

Alpha-lipoic acid. One randomized, double-blinded clinical trial that evaluated oral alpha-lipoic acid (ALA) for the prevention of platinum-induced peripheral neuropathy was identified. Patients received $600 \mathrm{mg}$ ALA acid 3 times daily for 24 weeks while receiving chemotherapy. This trial enrolled 243 patients, but only 70 of them (29\%) completed the trial. The study authors reported that the high dropout rate may have been related, in part, to the requirement that patients take the drug 3 times per day. Data indicated that neuropathy scores increased significantly from baseline for both groups at 24 weeks ( $P<.001$ for each group), with no statistically significant ameliorating effect from ALA in the treatment arm being observed from the Functional Assessment of Cancer Therapy/Gynecologic Oncology Group-Neurotoxicity (FACT/GOG-Ntx) tool, from pain scores, or from functional test scores. The study results suggest that ALA is not tolerated well and does not prevent neuropathy. ${ }^{17}$

Calcium and magnesium. One systematic review and 1 pilot trial not included in the systematic review evaluating the utility of intravenous calcium and magnesium were identified. The systemic review, which included 694 patients from 5 trials published between 2010 and 2014, confirmed that there was no beneficial effect in terms of the incidence of grade $\geq 2$ neuropathy (relative risk [RR], $0.81 ; 95 \% \mathrm{Cl}$, 0.60 to 1.11 ) or chronic neurotoxicity (RR, $0.95 ; 95 \% \mathrm{Cl}$, 0.69 to 1.32) from CaMg infusions for the prevention of oxaliplatin-induced peripheral neuropathy. ${ }^{18}$ Two older pooled analyses identified from Xu et $\mathrm{al}^{19}$ and Wen et a ${ }^{20}$, which did not come to the same conclusion, should be 


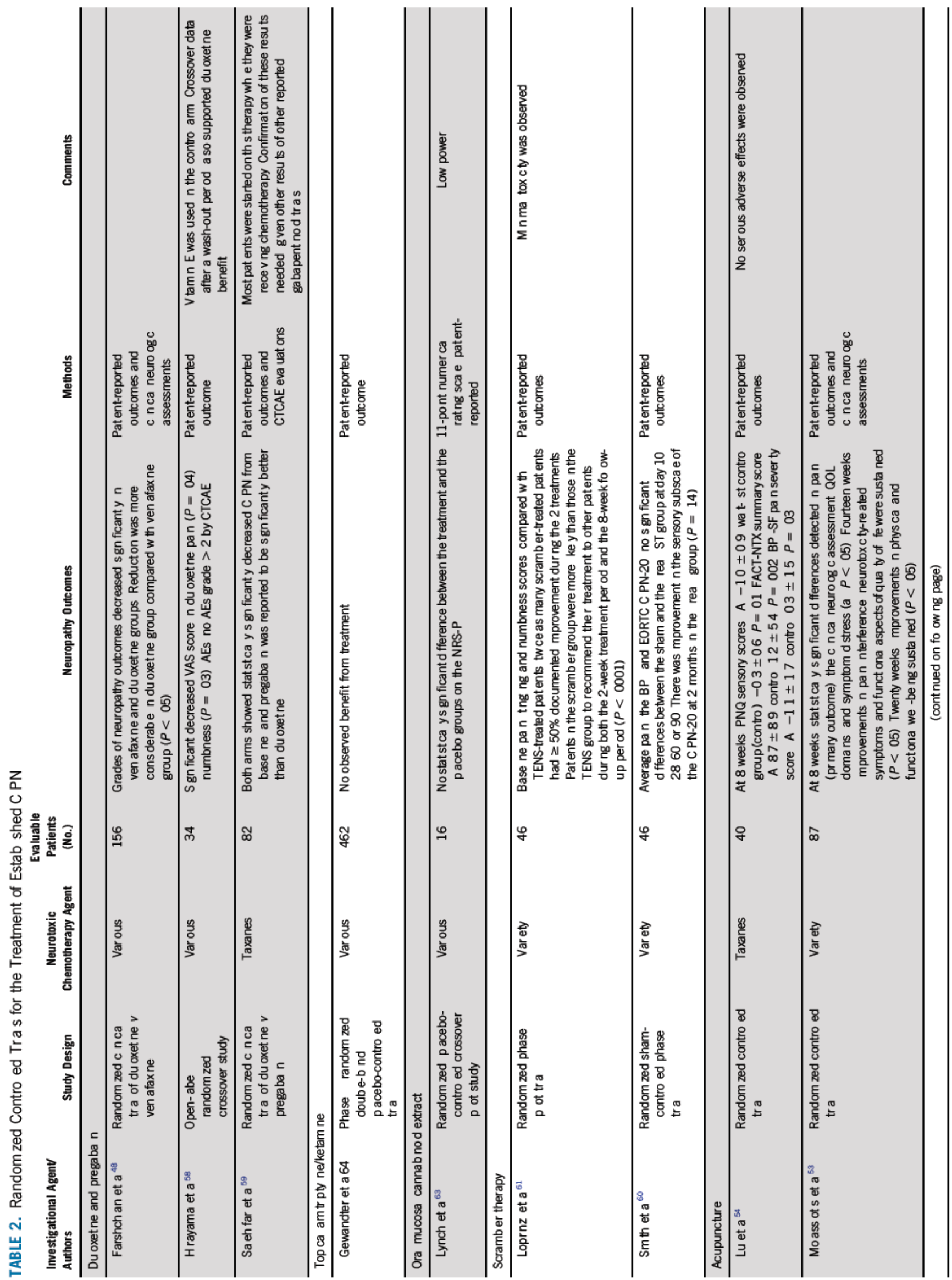




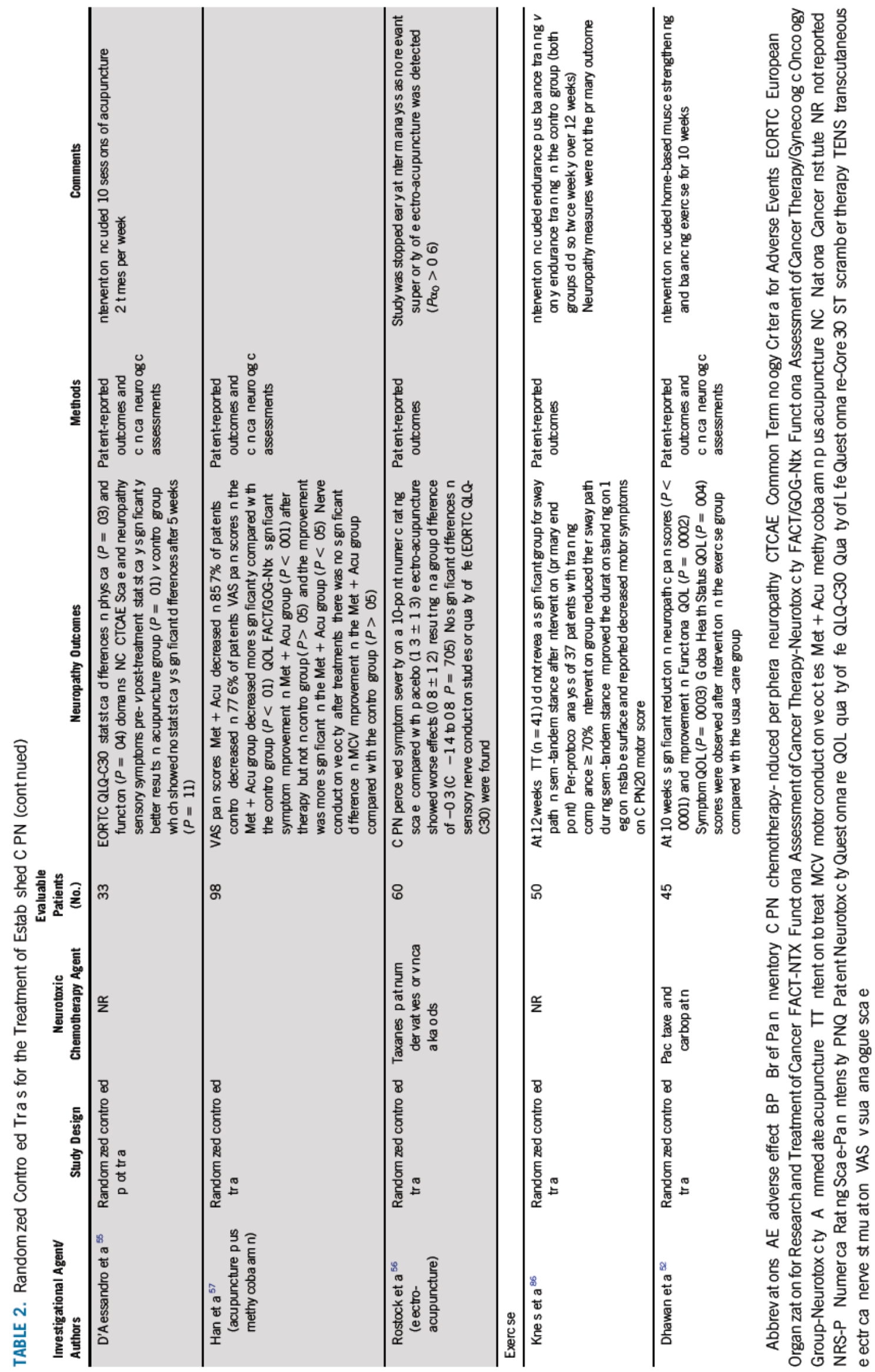


TABLE 3. Summary of Recommendations

\begin{tabular}{|c|c|c|c|c|}
\hline Interventions & Strength of Recommendation & Strength of the Evidence & Benefits & Harms $^{a}$ \\
\hline \multicolumn{5}{|l|}{ Prevention } \\
\hline Acetylcysteine & Moderate against & Intermediate & Low & Low \\
\hline Acupuncture & No recommendation & Low & Low & Moderate \\
\hline Amifostine & Moderate against & Intermediate & Low & Moderate \\
\hline Calcium and magnesium & Moderate against & Intermediate & Low & Low \\
\hline Cannabinoids & Moderate against & Intermediate & & \\
\hline Calmangafodipir & Moderate against & Intermediate & Low & Low \\
\hline Carbamazepine/oxcarbazepine & Moderate against & Intermediate & Low & Low \\
\hline L carnosine & Moderate against & Intermediate & Low & Low \\
\hline Exercise & No recommendation & Low & Low & Low \\
\hline Gabapentin/pregabalin & Moderate against & Intermediate & Low & Low \\
\hline GM 1 & No recommendation & Low & Low & Low \\
\hline Glutamate/glutamine & Moderate against & Intermediate & Low & Low \\
\hline GSH & Moderate against & Intermediate & Low & Low \\
\hline GJG Kampo medicine & Moderate against & Intermediate & Low & Low \\
\hline Metformin & Moderate against & Intermediate & Low & Low \\
\hline Minocycline & Moderate against & Intermediate & Low & Low \\
\hline Vitamin B & Moderate against & Intermediate & Low & Low \\
\hline Vitamin E & Moderate against & Intermediate & Low & Low \\
\hline \multicolumn{5}{|l|}{ Treatment } \\
\hline Acupuncture & No recommendation & Low & Low & Low \\
\hline Duloxetine & Moderate for & Intermediate & Moderate & Low \\
\hline Exercise & No recommendation & Low & Low & Low \\
\hline Gabapentin/pregabalin & No recommendation & Low & Low & Low \\
\hline BAK & No recommendation & Low & Low & Low \\
\hline Oral cannabinoids & No recommendation & Low & Low & Low \\
\hline Tricyclic antidepressants & No recommendation & Low & Low & Low \\
\hline Scrambler therapy & No recommendation & Low & Low & Low \\
\hline
\end{tabular}

Abbreviations: BAK, topical amitriptyline, ketamine, \pm baclofen; DDTC, diethyldithiocarbamate; GJG, goshajinkigan; GM 1, ganglioside monosialic acid; GSH, glutathione.

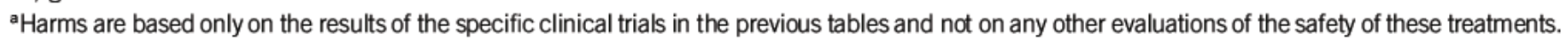


discounted, as they did not include data from the largest and most recent definitive trial.

A small randomized, placebo-controlled, crossover trial of calcium/magnesium for prevention of oxaliplatin-induced acute neuropathy involved 20 patients and evaluated EMG motor nerve hyperexcitability scores. ${ }^{21}$ The authors reported that there were no differences between those who received calcium and magnesium versus placebo in EMG outcomes (mean EMG score $\mathrm{Ca} / \mathrm{Mg}, 6.5$; standard deviation [SD], 4.31; and mean placebo score, 6.2; SD, 4.34) or for patient-reported acute neurotoxicity symptoms. ${ }^{21}$

Calmangafodipir. Calmangafodipir was studied in a placebo-controlled 3-arm phase II trial in patients receiving oxaliplatin-based chemotherapy. ${ }^{22}$ This trial provided promising enough data to initiate 2 phase III, placebo-controlled clinical trials (ClinicalTrials.gov identifiers: NCT04034355 and NCT04034355), with results forthcoming.

L-Carnosine. A 61-patient randomized trial evaluated a nutraceutical product, L-carnosine, as an agent to try to decrease oxaliplatin-induced neuropathy. ${ }^{23}$ Although the study reported remarkably positive results for the study agent over the control arm, there was no placebo used in this trial and it was not double-blinded. Clinicians judged neuropathy severity, as opposed to using patient-reported outcomes. Thus, additional data are necessary to understand the potential utility of this agent.

Cryotherapy/compression therapy/cryo-compression therapy. The first publication suggesting that cryotherapy was helpful for decreasing taxane-induced neuropathy came from Danish investigators, who noted that patients who received distal-extremity cryotherapy for decreasing onycholysis appeared to have reduced amounts of docetaxelinduced neuropathy by approximately $50 \%{ }^{24}$ Five trials evaluating cryotherapy were identified. One prospective, self-controlled trial in 36 patients with breast cancer treated weekly with paclitaxel, who wore frozen gloves (FGs) and socks on the dominant side for 90 minutes but not the other side, reported that the development of subjective CIPN symptoms was clinically and statistically significantly delayed during the course of the paclitaxel treatment; the occurrence of subjective CIPN at a cumulative dose of $960 \mathrm{mg} / \mathrm{m}^{2}$ was reported to be almost completely prevented (severe CIPN; hand: 2.8\% v 41.7\%; odds ratio [OR], infinite; $95 \% \mathrm{Cl}, 3.32$ to infinite; $P<.001$; foot: $2.8 \% v$ $36.1 \%$; OR, infinite; $95 \% \mathrm{Cl}, 2.78$ to infinite; $P<.001$ ), and the CIPN incidence, as assessed by other objective modalities, was lower on the intervention side. ${ }^{25}$ In a larger unblinded RCT, 180 patients started treatment with oxaliplatin, docetaxel, or paclitaxel and were randomly assigned to FGs on both hands during treatment or to usual care. ${ }^{26}$ Self-reported CIPN and QOL were measured. Overall neuropathy scores, the primary outcome measure, were not significantly different between the groups, in part because the feet were not treated, and neuropathy in lower extremities is oftentimes more problematic than it is in upper extremities. This study's results did support that FGs reduced neuropathy symptoms in patients' hands and improved some QOL measures. A recently published randomized phase II trial, involving 42 patients, compared cryotherapy (performed with ice packs on hands and feet) to an untreated control group who was not treated with cryotherapy. ${ }^{27}$ The area under the curve of the CIPN20 sensory scores over 12 weeks of paclitaxel was not found to differ between the study arms (mean difference, 3.45; $95 \% \mathrm{Cl}, \quad 3.13$ to $10.02 ; P \quad$.26). However, when the cryotherapy arm was compared with a control arm made up of controls combined from 3 previous trials, the cryotherapy arm had less neuropathy (Wilcoxon rank-sum $P \quad$.01). The authors of this study reported that the data supported phase III trial testing of this approach.

In a trial that evaluated a unilateral FG in 53 patients receiving docetaxel, $60 \%$ of the patients stopped the cryotherapy, and there were no differences between the hands that were randomly assigned to receive it versus not. ${ }^{28}$ Likewise, another study described similarly high drop-out rates and did not report positive findings. ${ }^{29}$

One trial evaluated continuous-flow limb hypothermia as a neuroprotective strategy in 20 patients receiving paclitaxel chemotherapy compared with usual care. Patients who received continuous limb hypothermia had less selfreported paclitaxel-induced neuropathy symptoms and had better nerve conduction studies. ${ }^{30}$ The same group of researchers also conducted a subsequent proof-of-concept study in patients with cancer receiving taxane chemotherapy. ${ }^{31}$ In this study, both cryotherapy and compression therapy (ie, cryo-compression therapy) were given to all 4 limbs in 13 subjects with each dose of paclitaxel. An analysis of nerve conduction studies with cryo-compression, administered at $16^{\circ} \mathrm{C}$ and a cyclic pressure of $5-15 \mathrm{~mm} \mathrm{Hg}$, illustrated preservation of motor amplitudes compared with baseline. ${ }^{32}$ In a cross-study comparison with their previous group of patients who had been treated with cryotherapy alone, patients appeared to do better with the combination therapy. ${ }^{32}$

One trial evaluated compression therapy using a tight surgical glove during taxane chemotherapy infusion. ${ }^{33}$ The intervention hand side was randomized within 43 patients. National Cancer Institute ( $\mathrm{NCl}$ ) Common Terminology Criteria for Adverse Events (CTCAE) grade $\geq 2$ sensory neuropathies were reported in $21 \%$ of the hands that wore the gloves versus $76 \%$ of hands that were not gloved.

A recently published small trial (38 patients) compared cryotherapy to compression therapy. This trial had patients use cryotherapy on one hand and compression therapy on the other and reported that similar results were seen with each approach. ${ }^{34}$ Additional randomized trials investigating cryotherapy and cryo-compression are ongoing.

Exercise. Three RCTs that evaluated various exercise interventions for the prevention of CIPN were identified. In 
a large trial of patients with cancer receiving taxane-, platinum-, or vinca alkaloid-based chemotherapy, ${ }^{35} 355$ patients were randomly assigned to chemotherapy or chemotherapy plus Exercise for Cancer Patients (EXCAP), a standardized, individualized, moderate-intensity, homebased, 6-week progressive walking and resistance exercise program. This unblinded trial was developed to evaluate the effectiveness of exercise on fatigue. As a secondary analysis, data regarding CIPN were also collected; these results supported that, compared with the control, exercise significantly reduced CIPN symptoms of hot/coldness in hands/feet $\left(\begin{array}{ll}P & .045\end{array}\right)$ and numbness and tingling, although the latter was not statistically significantly reduced compared with the control arm ( $P$.06). The intervention group still developed neuropathy, but less than the control group - a difference of approximately half a point on a 0-10 scale. On the basis of these findings and other preliminary supportive evidence, ${ }^{36,37}$ the $\mathrm{NCl}$ has recently approved a concept for a randomized cooperative oncology group trial to prospectively address the utility of exercise in this setting.

GM-1. GM-1 is a monosialo-glycosphingolipid that performs an important function in the processes of neurogenesis, nerve development and differentiation, cell recognition, and signal transduction. ${ }^{38}$ Two randomized trials investigating GM-1 for CIPN prevention were identified. In the first trial, Zhu et $\mathrm{al}^{39}$ reported on 120 patients with Gl cancers who were treated with oxaliplatin-based chemotherapy randomly assigned to receive intravenous ganglioside-monosialic acid or to a control group that received no neuroprotective agents. Although the grade of neurotoxicity in the experimental group was significantly lower than in the control group $(P<.05$, Mann-Whitney $U$ test), the lack of placebo control and the lack of patientreported outcome data decrease the confidence of this finding. ${ }^{39}$

The second trial was a placebo-controlled, double-blinded study of intravenous GM-1, given to prevent taxaneinduced CIPN in 183 patients with early-stage breast cancer. The study reported that treatment with GM-1 resulted in a statistically significant reduction in the severity and incidence of CIPN after 4 cycles of taxane-containing chemotherapy $(P<.001) .{ }^{38} \mathrm{~A}$ peculiar aspect of this trial is that the neuropathy appeared to be totally reversed in the placebo arm 3 months after chemotherapy completion, which is quite unusual in Western populations. ${ }^{40}$ Despite this very positive report, a confirmatory trial is needed.

Goshajinkigan. Our systematic review found a meta-analysis that pooled data from 5 trials and included 397 patients. The review reported that goshajinkigan was not associated with a reduced incidence of CIPN when assessed with the CTCAE (RR, 0.99; 95\% Cl, 0.53 to 1.85 for CIPN $\geq$ grade 2). ${ }^{41}$ Our systemic review did not find additional studies with goshajinkigan that were not included in this metaanalysis.
Metformin. One small randomized study ( $\mathrm{N}$ 40) that evaluated metformin as a means of preventing oxaliplatininduced neuropathy compared with a control group was identified. ${ }^{42}$ The authors reported that, at the end of the 12th FOLFOX-4 (fluorouracil, leucovorin, and oxaliplatin) regimen cycle, grade 2-3 neuropathy was lower in the metformin arm compared with the control arm $60 \% \mathrm{v}$ 95\%; $P \quad$.009), and the metformin arm had better NTX12 scores (24.0 v 19.2; $P<.001$ ). Given the small sample size, more confirmatory studies are needed before recommending this approach for oxaliplatin-induced neuropathy.

Gabapentin/pregabalin. Two randomized placebo-controlled trials investigating pregabalin were identified. On the basis of pilot study information, which suggested that gabapentinoids could decrease paclitaxel-associated acute pain and chronic neuropathy, investigators developed a phase II placebocontrolled clinical trial ( $\mathrm{N} 46$ ) to look at pregabalin for preventing these neuropathic problems. The results did not support that pregabalin was helpful for preventing the paclitaxel-associated acute pain syndrome or paclitaxelinduced peripheral neuropathy. ${ }^{43}$

In another double-blind, placebo-controlled trial, 143 painfree, chemotherapy-naive patients with colorectal cancer receiving at least 1 cycle of modified FLOX (ie, fluorouracil, leucovorin, and oxaliplatin) were randomly assigned to receive either pregabalin or placebo for 3 days before and 3 days after each oxaliplatin infusion. After following patients for up to 6 months, the authors reported that preemptive use of pregabalin during oxaliplatin infusions did not decrease the incidence of chronic pain related to oxaliplatin, measured by pain intensity and QOL scales. ${ }^{44}$ An additional randomized, double-blinded, placebo-controlled trial of pregabalin involving 64 patients who were receiving oxaliplatin chemotherapy was terminated early, as an interim analysis found that there were not sufficiently positive data to continue the trial. ${ }^{15}$

A small study (20 patients per arm) evaluating gabapentin 300 mg 3 times a day in a double-blind, randomized trial in patients receiving paclitaxel was identified. ${ }^{45}$ Although the authors reported a significant reduction in CIPN, confirmation of this is needed in a subsequent trial.

Venlafaxine. One trial investigating the efficacy of venlafaxine on prevention of CIPN was identified. Pursuant to data from Durand et $\mathrm{al}^{46}$ discussed in the initial ASCO CIPN guideline, this phase II randomized, placebo-controlled clinical trial was conducted to look at venlafaxine as a drug to decrease neuropathy associated with oxaliplatin. ${ }^{47}$ Fifty patients were randomly assigned to venlafaxine or placebo, given continuously with initiation of the first or second cycle of oxaliplatin. The trial results did not support the use of venlafaxine in this setting, dampening enthusiasm for proceeding with a phase III trial. ${ }^{47}$ Notably, the Durand et $\mathrm{al}^{46}$ study started venlafaxine/placebo after 
patients had received some oxaliplatin, in contrast to at oxaliplatin initiation. Given that there are now data that support that venlafaxine may decrease symptoms in patients with established neuropathy (although not as well as duloxetine), ${ }^{48}$ it may be that in the Durand et $\mathrm{al}^{46}$ trial venlafaxine was potentially acting as an agent that treated established neuropathy, as opposed to acting as a prevention agent.

Vitamin B. A 71-patient placebo-controlled 2-arm trial evaluated an oral vitamin B product in patients who were receiving a variety of neurotoxic drugs (taxanes, oxaliplatin, or vincristine). ${ }^{49}$ Data were only available for 47 patients and, understandably with this small sample size and the variety of chemotherapy drugs, there was no suggestion that the primary end point was improved in the vitamin B arm.

Vitamin E. One systemic review and meta-analysis plus another trial not included in the meta-analysis were identified. The systematic review and meta-analysis of 6 studies that included 353 patients reported that the administration of vitamin $E$ (at doses that included $300 \mathrm{mg}$ daily, $300 \mathrm{mg}$ twice daily, and $400 \mathrm{mg}$ daily) did not decrease the incidence of CIPN (RR, 0.55; 95\% Cl, 0.29 to 1.05; $P$ $.07 .{ }^{50}$ The small study published subsequently to the metaanalysis $^{51}$ also concluded that vitamin $\mathrm{E}$ did not help to prevent oxaliplatin-induced peripheral neuropathy.

Clinical interpretation regarding efforts to prevent CIPN. The current review did not find studies supporting the recommendation of any neuropathy-preventative agent. Unlike the promising original guideline commentary regarding venlafaxine as a preventative agent, the updated guideline does not recommend it. A negative follow-up study with a similar number of patients, which treated patients for a longer time period and used a more accepted chemotherapy neuropathy patient-reported outcome measurement tool, backs this. ${ }^{47}$

Given the dearth of effective established agents for preventing chemotherapy-induced neuropathy and the limited effective therapy for treating established CIPN, patients/ clinicians should weigh the benefits of using neuropathyinducing agents against the risks of developing long-term, irreversible CIPN.

Although proof of benefit has not been established, available data support that exercise, cryotherapy, compression therapy, and/or cryo-compression therapy may, in part, prevent CIPN symptoms and appear to be reasonably safe, although clinicians and patients should be aware of frostbite risk. Ganglioside-monosialic acid seemed to be effective in preventing taxane-induced peripheral neuropathy in Chinese patients, but this should be confirmed in a large trial in a different ethnic group. Ongoing trials are attempting to better define whether one or more of these methods will safely prevent CIPN.

\section{Treatment of Chemotherapy-Induced Peripheral Neuropathy That Develops While Patients Are Receiving Neurotoxic Chemotherapy}

2.1 Clinicians should assess, and discuss with patients, the appropriateness of dose delaying, dose reduction, or stopping chemotherapy (or substituting with agents that do not cause CIPN) in patients who develop intolerable neuropathy and/or functional nerve impairment (Type of recommendation: informal consensus, benefits outweigh harms; Evidence quality: low; Strength of recommendation: moderate).

Clinical interpretation. Although there are limited clinical trial data available to guide practice when patients develop CIPN during the course of neurotoxic chemotherapy, this is a common clinical practice situation. Scenarios vary from patients who are being treated with curative intent versus palliative-intent chemotherapy for advanced cancer. Clinicians and patients may make different decisions for continuing neurotoxic chemotherapy in patients suffering from significant neuropathy, based on whether the patient is receiving adjuvant chemotherapy that might improve survival probabilities by a percentage point or two, versus a patient receiving adjuvant chemotherapy expected to improve survival probabilities by many percentage points, versus a patient with metastatic disease. In these individual situations, clinicians may determine whether to reasonably use alternative chemotherapy regimens that do not cause neurotoxicity. Clinicians should obtain individual patient perspectives in all these situations.

\section{Treatment of Chemotherapy-Induced Peripheral Neuropathy for Patients Who Have Completed Neurotoxic Chemotherapy}

3.1 For patients with cancer experiencing painful CIPN, clinicians may offer duloxetine (Type of recommendation: evidence based, benefits equal harms; Evidence quality: intermediate; Strength of recommendation: moderate).

3.2 Outside the context of a clinical trial, no recommendations can be made on the use of the following interventions for the treatment of CIPN:

- Exercise therapy

- Acupuncture

- Scrambler therapy

- Gabapentin/pregabalin

- Topical gel treatment containing baclofen, amitriptyline HCL, plus/minus ketamine

- Tricyclic antidepressants

- Oral cannabinoids

(Type of recommendation: no recommendation; Evidence quality: low; Strength of recommendation: not applicable).

Note: While recent preliminary evidence suggests a potential for benefit from exercise, acupuncture, and scrambler therapy, larger sample-sized definitive studies are needed to confirm efficacy and clarify risks. 


\section{Literature Review Update and Analysis for Treatment of CIPN}

Exercise. Current data from an RCT mildly suggest that exercise is a feasible, safe, and promising supportive measure for patients with cancer experiencing CIPN. The trial randomly assigned 45 patients with established CIPN to a 10-week home-based muscle strengthening and balancing exercise program versus usual care. The patients in the exercise group experienced a significant reduction in neuropathic pain scores $(P<.0001)$ and improvement in Functional QOL $\left(\begin{array}{ll}P & .0002)\end{array}\right.$ Symptom QOL ( $P$.0003) and Global Health Status QOL ( $P$ .004) compared with those randomly assigned to the usualcare group. $^{52}$ The lack of an active control group diminishes the strength of the findings. Another small trial evaluated patients with metastatic colorectal cancer randomly assigned to an exercise program versus a wait-list control group. ${ }^{36}$ Those receiving exercise had relatively stable CIPN scores over time, while the wait-list control group's CIPN worsened.

Acupuncture. Five trials evaluating the efficacy of acupuncture for the treatment of CIPN were identified, ${ }^{53-55}$ including 1 trial that evaluated electro-acupuncture ${ }^{56}$ and another that evaluated acupuncture combined with methylcobalamin. ${ }^{57} \mathrm{~A}$ randomized assessor-only-blinded controlled trial of acupuncture twice weekly for 8 weeks versus a wait-list control group involving 87 patients with cancer reported significant changes at 8 weeks in pain measured using the Brief Pain Inventory (BPI). ${ }^{53}$ Significant improvements in clinical neurologic assessment, QOL domains, and symptom distress were also reported (all $P<$.05). Improvements in pain interference, neurotoxicity-related symptoms, and functional aspects of QOL were sustained in a 14-week assessment $(P<.05)$, as were physical and functional well-being at a 20-week assessment $(P<.05)$.

A pilot trial involving 40 women with stage I-III breast cancer and grade $\geq 1$ CIPN after taxane-containing adjuvant chemotherapy investigated immediate acupuncture versus a wait-list control. ${ }^{54}$ At 8 weeks, participants in the treatment arm experienced significant improvements in the Patient Neurotoxicity Questionnaire (PNQ) sensory score $\left(\begin{array}{ll}P & .01\end{array}\right)$, FACT-NTX summary score ( $P$.002), and BPI-Short Form pain severity score $P \quad$.03) compared with those in the control arm. No serious adverse effects were observed.

Another pilot trial randomly assigned 33 adult patients with cancer and CIPN into 2 groups (control and acupuncture: treated with 10 sessions, twice a week). ${ }^{55}$ Statistically significant differences were reported in physical $\left(\begin{array}{ll}P & .03\end{array}\right)$ and function ( $P$.04) domains of the European Organization for Research and Treatment of Cancer (EORTC) Quality of Life Questionnaire-Core 30 when comparing between control and acupuncture groups. $\mathrm{NCl}$ CTCAE Scale and neuropathy sensory symptoms were also improved in the acupuncture group between pretreatment and 5 weeks post-treatment $(P \quad .01)$, whereas no such differences were detected in the control group $\left(\begin{array}{ll}P & .11\end{array}\right)$.
The use of electro-acupuncture was not superior to placebo in a randomized trial of 59 patients with CIPN. ${ }^{56}$ The trial failed to show efficacy compared with placebo, as determined by using a predefined statistical threshold at the first interim analysis.

Another trial in 98 patients compared acupuncture combined with methylcobalamin to methylcobalamin alone and found that after 3 cycles of therapy the pain was significantly mitigated in the methylcobalamin plus acupuncture group. ${ }^{57}$ Visual analogue scale (VAS) pain scores decreased more in the methylcobalamin plus acupuncture group than the methylcobalamin control group $(P<.01)$.

Duloxetine and pregabalin. Two duloxetine trials were published after the initial ASCO CIPN guideline publication. ${ }^{48,58}$ One trial randomly assigned patients with CIPN to 3 pharmacotherapy groups: venlafaxine, duloxetine, and placebo. ${ }^{48}$ The authors reported decreased neuropathy in the venlafaxine and duloxetine groups, with a better reduction in the duloxetine group compared with venlafaxine group $(P<.05)$. In another open-label, randomized, crossover study, 34 patients with cancer were randomly assigned to receive duloxetine $(20 \mathrm{mg} / \mathrm{d}$ orally for the first week and $40 \mathrm{mg} / \mathrm{d}$ for the next 3 weeks) or vitamin B12 ( $1.5 \mathrm{mg} / \mathrm{d}$ orally for 4 weeks). ${ }^{58}$ After a 2 - to 4-week washout period, treatment was crossed over for another 4 weeks. Decreases in the mean VAS scores for numbness and pain were seen during the periods of duloxetine administration. Significant differences were observed between the duloxetine-first and the vitamin B12-first groups with respect to numbness $\left(\begin{array}{lll}P & .03\end{array}\right)$ and pain $\left(\begin{array}{ll}P & .04\end{array}\right)$ at 4 weeks after administration.

In January 2020, a trial was published that randomly assigned patients with paclitaxel- or docetaxel-associated CIPN to receive duloxetine versus pregabalin, with 40-42 patients per arm. They reported $a \geq 33 \%$ improvement of visual analog scores in the duloxetine and pregabalin arms at 6 weeks of $38 \%$ and $93 \%$, respectively $(P<.001) .{ }^{59}$ The majority of the patients in both arms started their treatment while they were receiving chemotherapy, and some of this improvement may have been related to chemotherapy discontinuation.

Scrambler therapy. Two randomized trials evaluating scrambler therapy, an electrocutaneous treatment approach, were found..$^{60,61}$ One randomized sham-controlled phase II trial in 33 patients who received 30-minute sessions of scrambler therapy (ST) or sham treatment found no significant differences between the sham and the experimental ST group for BPI average pain or the EORTC CIPN-20. ${ }^{60}$

The second phase II trial randomly assigned patients with CIPN symptoms for at least 3 months to receive ST or transcutaneous electrical nerve stimulation (TENS) for 2 weeks. In 46 evaluable patients, twice as many ST-treated patients had at least a 50\% documented improvement during the 2 treatment weeks from their baseline pain, tingling, and numbness scores when compared with the TENS-treated patients (from 36\%-56\% compared with 
16\%-28\% for each symptom). ${ }^{61}$ Global Impression of Change scores for "neuropathy symptoms," pain, and QOL improved similarly. Moreover, patients in the ST group were more likely than those in the TENS group to recommend their treatment to other patients, during both the 2-week treatment period and the 8-week follow-up period $(P<.0001){ }^{61}$ The publication did not report any substantial adverse events. ${ }^{62}$

Oral mucosal cannabinoid extract. A small, randomized, placebo-controlled clinical trial of 18 patients evaluated the role of nabiximols, an oral mucosal cannabinoid spray, for chemotherapy-induced neuropathic pain. ${ }^{63}$ In this crossover clinical trial, 16 of the 18 randomly assigned patients completed the study. Noting the small number of patients, there was no suggestion of differential benefits in neuropathy scores between the active and placebo agents. Yet, there was more evidence of toxicity (fatigue, dry mouth, dizziness, and nausea) in the patients receiving the cannabinoid preparation, decreasing interest in this approach.

Topical amitriptyline/ketamine. A topical $4 \%$ amitriptyline/ $2 \%$ ketamine preparation was studied as a treatment of established chemotherapy neuropathy in a randomized, placebo-controlled trial involving 462 patients. ${ }^{64}$ Patients with average 7-day pain, numbness, and tingling ratings of at least 4 on an 11-point numeric rating scale were eligible for enrollment in the study. Topical amitriptyline/ketamine showed no effect on 6-week CIPN scores (adjusted mean difference, $\quad 0.17 ; P \quad$.363), and this trial did not support that using this topical preparation alleviated chemotherapyinduced pain, numbness, or tingling.

Clinical interpretation regarding the treatment of established CIPN. Additional data, which have become available since the previous ASCO CIPN guideline, further support the utility of duloxetine for treating established painful CIPN. Conversely, there have not been any further clinical trials to strongly support the utility of tricyclic antidepressants, gabapentinoids, or topical amitriptyline/ketamine/baclofen, decreasing the tepid support that was provided for these 3 therapeutic approaches in the initial ASCO CIPN guideline. In addition, newer published reports do not provide support for a topical amitriptyline/ketamine preparation or an oral mucosal cannabinoid product.

Although proof of benefit has not been provided, data suggestive of benefit support that 3 approaches (scrambler therapy, acupuncture, and exercise) may diminish established CIPN symptoms and appear to be reasonably safe. Further research is needed to better delineate the utility, or its lack thereof, of these approaches in treating established CIPN.

\section{DISCUSSION}

The current review found no additional studies supporting the use of any preventative approach for neuropathy. In contrast with the promising original guideline commentary regarding venlafaxine as a preventative agent, longer followup data do not support its use. ${ }^{47}$
For treatment of established painful neuropathy, duloxetine remains the sole recommended treatment. Along with the data demonstrating that duloxetine decreases CIPN pain, there is a suggestion from exploratory analyses that it also decreases nonpainful CIPN symptoms. ${ }^{58,65}$ When patients stop duloxetine, it should be tapered slowly, as stopping abruptly can lead to withdrawal symptoms.

Acetyl-L-carnitine data were inconclusive for the treatment of established neuropathy at the time of the initial ASCO guideline publication. A new larger trial reported that there was no benefit for acetyl-L-carnitine for treating chemotherapyinduced neuropathy. Consequently, the current updated guideline recommends against acetyl-L-carnitine for the treatment of established chemotherapy-induced neuropathy. ${ }^{14}$

There were 3 treatments that were inconclusive in the original guideline but "reasonable to try in some situations," namely tricyclic antidepressants, gabapentinoids, and a topical gel treatment containing baclofen, amitriptyline, and ketamine. Although data regarding these 3 treatment options remain inconclusive, there is waning enthusiasm regarding them.

Regarding the tricyclic antidepressants, the previous guideline indicated that tricyclic antidepressant use was reasonable to try, primarily on the basis of their utility in other neuropathy situations, but not on the basis of any positive randomized clinical trials demonstrating any utility of this drug class for treating established CIPN. Currently, the use of tricyclic antidepressants does not appear to be common, because of their lack of established benefit and/ or their unfavorable side effects.

Regarding topical baclofen, amitriptyline, and ketamine, the previous guideline noted that a placebo-controlled trial was promising. However, there are reasons to be less enthusiastic about this approach now: (1) no additional trials have been conducted; (2) there is not an US Food and Drug Administration-approved product available, and the only way to get this treatment is to have it compounded; and (3) there was a subsequent publication of a negative trial that studied topical amitriptyline and ketamine. ${ }^{64}$ However, the lack of baclofen in this latter preparation may explain the negative finding of the study.

The suggestion in the initial ASCO CIPN guideline that gabapentinoids might be helpful and worth trying for chemotherapy-induced neuropathy was also primarily based on gabapentinoid efficacy against other types of neuropathies, like diabetic neuropathy. Presently, this endorsement is harder to support. With the 1 older placebocontrolled clinical trial that showed no benefit for gabapentin for the treatment of chemotherapy-induced peripheral neuropathy, ${ }^{66} 2$ subsequent trials investigating pregabalin as an agent to prevent chemotherapy-induced neuropathy ( 1 for paclitaxe ${ }^{43}$ and 1 for oxaliplatin [ClinicalTrials.gov Identifier: NCT00380874]) failed to provide evidence of benefit. Although prevention trials are certainly 
different from treatment trials, if pregabalin was given continuously while the patient developed neuropathy in a prevention trial, one would have expected to see a decrease in the severity of neuropathic symptoms if it was truly beneficial for treating established neuropathy. In contrast to these negative gabapentinoid data, 1 trial $^{59}$ suggests that pregabalin was helpful. Confirmation of these data is necessary before endorsement of routine use of gabapentinoids for treating established CIPN.

Historically, the first known report on using gabapentin for chemotherapy-induced neuropathy came from Italian authors at the 2000 ASCO annual meeting, entitled "Oxaliplatin-induced Neuropathy: Could Gabapentin be the Answer?"67 This report describes the use of gabapentin in 7 patients who developed neuropathy while receiving oxaliplatin. With the initiation of neuropathy, gabapentin was given at $100 \mathrm{mg}$ twice per day. Clinicians could increase gabapentin to $100 \mathrm{mg} 3$ times daily if the lower daily dose did not resolve symptoms. The abstract reported there was a disappearance of neuropathy symptoms, which continued even with the use of up to 14 total oxaliplatin doses. This work is not available in manuscript form. In retrospect, it does not seem biologically plausible that this very low dose of gabapentin (given that target doses of this drug can be $\geq 3,000 \mathrm{mg} / \mathrm{d}$ ) could have had such a dramatic benefit. A body of other published articles regarding gabapentin for treating CIPN (ranging from case reports to case series to 1 randomized placebo-controlled trial) $)^{66,6874} \mathrm{do}$ not, on the whole, support the utility of gabapentin for treating established CIPN.

Notably, some insurance companies require that patients with CIPN receive a gabapentinoid agent before allowing the use of duloxetine. ${ }^{75}$ Additional support for this contention comes from a recent article reporting that on insurance claims data the use of gabapentinoids (gabapentin or pregabalin) was more than 8-fold higher than was the use of duloxetine in patients who had recently received neurotoxic chemotherapy. ${ }^{76}$ This contradicts the recommendations of the previous and current ASCO CIPN guidelines.

Although the current guideline is primarily focused on means of preventing CIPN and/or treating established CIPN, CIPN can involve physical dysfunction; patients with CIPN have balance troubles and a higher chance of falling. ${ }^{77}$ Therefore, it is reasonable to consider physical therapy and/or occupational therapy approaches for patients with such CIPN-related disabilities.

A summary of the recommendations is provided in Table 3.

\section{GUIDELINE IMPLEMENTATION}

ASCO guidelines are developed for implementation across health settings. Each ASCO guideline includes a member from ASCO's Practice Guideline Implementation Network (PGIN) on the panel. The additional role of this PGIN representative on the guideline panel is to assess the suitability of the recommendations to implementation in the community setting and also to identify any other barrier to implementation a reader should be aware of. Barriers to implementation include the need to increase awareness of the guideline recommendations among front-line practitioners and survivors of cancer and caregivers and also to provide adequate services in the face of limited resources. The guideline Bottom Line Box was designed to facilitate implementation of recommendations. This guideline will be distributed widely through the ASCO PGIN. ASCO guidelines are posted on the ASCO website and most often published in Journal of Clinical Oncology and Journal of Oncology Practice.

\section{LIMITATION OF THE RESEARCH AND FUTURE RESEARCH}

Inconsistent subjective and objective outcome measures, choice of control group, and duration of exposure have resulted in challenges in interpreting some of the prior studies. $\mathrm{NCl}$-sponsored studies are ongoing to better define the phenotype of CIPN, to ensure consistency in outcome measures from study to study going forward.

Better interventions are needed to prevent CIPN. Ongoing and planned trials will, likely, better clarify the role of exercise, compression therapy, cryotherapy, and other targeted interventions. Several planned and/or ongoing preclinical studies are evaluating the role of neuronal transport, neuroprotection, neuro-inflammation, serotonin-norepinephrine reuptake, nociceptor sodium channel inhibition, mitochondrial enzymes, and oxidative stress. ${ }^{79-81}$ Many of the above agents target DNA damage related to inflammation, reactive oxygen species, and oxidative stress, supporting this as a thematic target for prevention of CIPN.

Better agents are also needed to treat established CIPN. Ongoing and planned clinical trials should better clarify the role of exercise, acupuncture, scrambler therapy, and other targeted interventions. Topical therapies such as capsaicin might also be further explored. ${ }^{82}$

Clinicaltrials.gov currently lists $>100$ clinical trials related to CIPN that are actively accruing patients or in development. We hope that results from these trials will lead to new means of preventing and/or treating CIPN.

\section{ADDITIONAL RESOURCES}

More information, including a supplement with additional evidence tables, slide sets, and clinical tools and resources, is available at www.asco.org/survivorship-guidelines. Patient information is available at www.cancer.net.

RELATED ASCO GUIDELINES

- Patient-Clinician Communication ${ }^{83}$ (http:// ascopubs.org/doi/10.1200/JC0.2017.75.2311) 


\section{AFFILIATIONS}

${ }^{1}$ Mayo Clinic, Rochester, MN

${ }^{2}$ American Society of Clinical Oncology, Alexandria, VA

${ }^{3}$ Sanford USD Medical Center, Sioux Falls, SD

${ }^{4}$ University of Milano Bicocca, Monza, Italy

${ }^{5}$ Wichita, KS

${ }^{6}$ University of Michigan, Ann Arbor, MI

${ }^{7}$ Indiana University School of Medicine, Indianapolis, IN

${ }^{8}$ Mass General North Shore Cancer Center, Danvers, MA

${ }^{9}$ Ohio State University, Columbus, $\mathrm{OH}$

${ }^{10}$ Northwestern University, Chicago, IL

${ }^{11}$ Indiana University School of Medicine, Indianapolis, IN

${ }^{12}$ Research Advocacy Network, Plano, TX

${ }^{13}$ Johns Hopkins, Baltimore, MD

${ }^{14}$ Columbia University Medical Center, New York, NY

\section{CORRESPONDING AUTHOR}

American Society of Clinical Oncology, 2318 Mill Rd, Suite 800, Alexandria, VA 22314; e mail: guidelines@asco.org.

\section{EQUAL CONTRIBUTION}

C.L.L. and D.L.H. were Expert Panel co chairs

\section{EDITOR'S NOTE}

This American Society of Clinical Oncology (ASCO) Clinical Practice Guideline provides recommendations, with comprehensive review and analyses of the relevant literature for each recommendation. Additional information, including a supplement with additional evidence tables, slide sets, clinical tools and resources, and links to patient information at www.cancer.net, is available at www.asco.org/supportive care guidelines.

\section{AUTHOR CONTRIBUTIONS}

Conception and design: Charles L. Loprinzi, Christina Lacchetti, Cynthia Chauhan, Mark R. Kelley, Ellen M. Lavoie Smith, Thomas J. Smith, Dawn L. Hershman

Administrative support: Christina Lacchetti

Collection and assembly of data: Charles L. Loprinzi, Christina Lacchetti, Thomas J. Smith, Dawn L. Hershman

Data analysis and interpretation: Charles L. Loprinzi, Jonathan Bleeker, Guido Cavaletti, Daniel L. Hertz, Antoinette Lavino, Maryam B. Lustberg, Judith A. Paice, Bryan P. Schneider, Ellen M. Lavoie Smith, Mary Lou Smith, Thomas J. Smith, Nina Wagner Johnston, Dawn L. Hershman Manuscript writing: All authors

Final approval of manuscript: All authors Accountable for all aspects of the work: All authors

\section{AUTHORS' DISCLOSURES OF POTENTIAL CONFLICTS OF INTEREST AND DATA AVAILABILITY STATEMENT}

Disclosures provided by the authors and data availability statement (if applicable) are available with this article at DOI https://doi.org/10.1200/ JC0.20.01399.

\section{ACKNOWLEDGMENT}

We thank Ting Bao, MD, DABMA, MS, Zoneddy Dayao, MD, Emily Tonorezos, MD, MPH, and Jørn Herrstedt MD, DMSc, and the Clinical Practice Guidelines Committee for their thoughtful reviews and insightful comments on this guideline. All Appendices and Acknowledgment material (including the table of Expert Panel members) are online only. They will appear on JCO website online but not in the print version.

\section{REFERENCES}

1. Reeves BN, Dakhil SR, Sloan JA, et al: Further data supporting that paclitaxel associated acute pain syndrome is associated with development of peripheral neuropathy: North Central Cancer Treatment Group trial N08C1. Cancer 118:5171 5178, 2012

2. Loprinzi CL, Reeves BN, Dakhil SR, et al: Natural history of paclitaxel associated acute pain syndrome: Prospective cohort study NCCTG N08C1. J Clin Oncol 29:1472 1478, 2011

3. Pachman DR, Qin R, Seisler DK, et al: Clinical course of oxaliplatin induced neuropathy: Results from the randomized phase III trial N08CB (Alliance). J Clin Oncol 33:3416 3422, 2015

4. Ruddy KJ, Pachman D, Qin R, et al: A comparison of the natural history of oxaliplatin and paclitaxel induced neuropathy. (NCCTG N08C1, N08CB/Alliance). J Clin Oncol 33:9564 9564, 2015

5. Loprinzi CL, Maddocks Christianson K, Wolf SL, et al: The paclitaxel acute pain syndrome: Sensitization of nociceptors as the putative mechanism. Cancer J 13: 399 403, 2007

6. Bandos H, Melnikow J, Rivera DR, et al: Long term peripheral neuropathy in breast cancer patients treated with adjuvant chemotherapy: NRG Oncology/ NSABP B 30. J Natl Cancer Inst 110:djx162, 2018

7. Hershman DL, Unger JM, Crew KD, et al: Two year trends of taxane induced neuropathy in women enrolled in a randomized trial of acetyl $L$ carnitine (SWOG S0715). J Natl Cancer Inst 110:669 676, 2018

8. Alberti P, Rossi E, Argyriou AA, et al: Risk stratification of oxaliplatin induced peripheral neurotoxicity applying electrophysiological testing of dorsal sural nerve. Support Care Cancer 26:3143 3151, 2018

9. Velasco R, Bruna J, Briani C, et al: Early predictors of oxaliplatin induced cumulative neuropathy in colorectal cancer patients. J Neurol Neurosurg Psychiatry 85:392 398, 2014

10. Chen X, Stubblefield MD, Custodio CM, et al: Electrophysiological features of taxane induced polyneuropathy in patients with breast cancer. J Clin Neurophysiol 30:199 203, 2013

11. Shojania KG, Sampson M, Ansari MT, et al: How quickly do systematic reviews go out of date? A survival analysis. Ann Intern Med 147:224 233, 2007

12. Shea BJ, Reeves BC, Wells G, et al: AMSTAR 2: A critical appraisal tool for systematic reviews that include randomised or non randomised studies of healthcare interventions, or both. BMJ 358:j4008, 2017

13. Greenlee H, Crew KD, Capodice J, et al: Randomized sham controlled pilot trial of weekly electro acupuncture for the prevention of taxane induced peripheral neuropathy in women with early stage breast cancer. Breast Cancer Res Treat 156:453 464, 2016

14. Campone M, Berton Rigaud D, Joly Lobbedez F, et al: A double blind, randomized phase II study to evaluate the safety and efficacy of acetyl $L$ carnitine in the prevention of sagopilone induced peripheral neuropathy. Oncologist 18:1190 1191, 2013

15. Reference deleted

16. Hershman DL, Unger JM, Crew KD, et al: Randomized double blind placebo controlled trial of acetyl $L$ carnitine for the prevention of taxane induced neuropathy in women undergoing adjuvant breast cancer therapy. J Clin Oncol 31:2627 2633, 2013 
17. Guo Y, Jones D, Palmer JL, et al: Oral alpha lipoic acid to prevent chemotherapy induced peripheral neuropathy: A randomized, double blind, placebo controlled trial. Support Care Cancer 22:1223 1231, 2014

18. Jordan B, Jahn F, Beckmann J, et al: Calcium and magnesium infusions for the prevention of oxaliplatin induced peripheral neurotoxicity: A systematic review. Oncology 90:299 306, 2016

19. Xu XT, Dai ZH, Xu Q, et al: Safety and efficacy of calcium and magnesium infusions in the chemoprevention of oxaliplatin induced sensory neuropathy in gastrointestinal cancers. J Dig Dis 14:288 298, 2013

20. Wen F, Zhou Y, Wang W, et al: Ca/Mg infusions for the prevention of oxaliplatin related neurotoxicity in patients with colorectal cancer: A meta analysis. Ann Oncol 24:171 178, 2013

21. Han $\mathrm{CH}$, Khwaounjoo P, Kilfoyle DH, et al: Phase I drug interaction study of effects of calcium and magnesium infusions on oxaliplatin pharmacokinetics and acute neurotoxicity in colorectal cancer patients. BMC Cancer 13:495, 2013

22. Glimelius B, Manojlovic N, Pfeiffer $P$, et al: Persistent prevention of oxaliplatin induced peripheral neuropathy using calmangafodipir (PledOx): A placebo controlled randomised phase II study (PLIANT). Acta Oncol 57:393 402, 2018

23. Yehia R, Saleh S, El Abhar H, et al: L carnosine protects against oxaliplatin induced peripheral neuropathy in colorectal cancer patients: A perspective on targeting Nrf 2 and NF кB pathways. Toxicol Appl Pharmacol 365:41 50, 2019

24. Eckhoff L, Knoop AS, Jensen MB, et al: Risk of docetaxel induced peripheral neuropathy among 1,725 Danish patients with early stage breast cancer. Breast Cancer Res Treat 142:109 118, 2013

25. Hanai A, Ishiguro H, Sozu T, et al: Effects of cryotherapy on objective and subjective symptoms of paclitaxel induced neuropathy: prospective self controlled trial. J Natl Cancer Inst 110:141 148, 2018

26. Beijers AJM, Bonhof CS, Mols F, et al: Multicenter randomized controlled trial to evaluate the efficacy and tolerability of frozen gloves for the prevention of chemotherapy induced peripheral neuropathy. Ann Oncol 31:131 136, 2020

27. Ruddy KJ, Le Rademacher J, Lacouture ME, et al: Randomized controlled trial of cryotherapy to prevent paclitaxel induced peripheral neuropathy (RU221511I); an ACCRU trial. Breast 48:89 97, 2019

28. McCarthy AL, Shaban RZ, Gillespie K, et al: Cryotherapy for docetaxel induced hand and nail toxicity: randomised control trial. Support Care Cancer 22: 1375 1383, 2014

29. Griffiths C, Kwon N, Beaumont JL, et al: Cold therapy to prevent paclitaxel induced peripheral neuropathy. Support Care Cancer 26:3461 3469, 2018

30. Sundar R, Bandla A, Tan SS, et al: Limb hypothermia for preventing paclitaxel induced peripheral neuropathy in breast cancer patients: A pilot study. Front Oncol 6:274, 2017

31. Bandla A, Tan S, Kumarakulasinghe NB, et al: Safety and tolerability of cryocompression as a method of enhanced limb hypothermia to reduce taxane induced peripheral neuropathy. Support Care Cancer 28:3691 3699, 2020

32. Sundar R, Bandla A, Tan S, et al: Cryocompression for enhanced limb hypothermia in preventing paclitaxel induced peripheral neuropathy. J Clin Oncol 36, 2018 (15 suppl; abstr 10095)

33. Tsuyuki S, Senda N, Kanng Y, et al: Evaluation of the effect of compression therapy using surgical gloves on nanoparticle albumin bound paclitaxel induced peripheral neuropathy: A phase II multicenter study by the Kamigata Breast Cancer Study Group. Breast Cancer Res Treat 160:61 67, 2016

34. Kanbayashi Y, Sakaguchi K, Ishikawa T, et al: Comparison of the efficacy of cryotherapy and compression therapy for preventing nanoparticle albumin bound paclitaxel induced peripheral neuropathy: A prospective self controlled trial. Breast 49:219 224, 2020

35. Kleckner IR, Kamen C, Gewandter JS, et al: Effects of exercise during chemotherapy on chemotherapy induced peripheral neuropathy: A multicenter, randomized controlled trial. Support Care Cancer 26:1019 1028, 2018

36. Zimmer P, Trebing S, Timmers Trebing $U$, et al: Eight week, multimodal exercise counteracts a progress of chemotherapy induced peripheral neuropathy and improves balance and strength in metastasized colorectal cancer patients: A randomized controlled trial. Support Care Cancer 26:615 624, 2018

37. Courneya KS, McKenzie DC, Mackey JR, et al: Subgroup effects in a randomised trial of different types and doses of exercise during breast cancer che motherapy. Br J Cancer 111:1718 1725, 2014

38. Su Y, Huang J, Wang S, et al: The effects of ganglioside monosialic acid in taxane induced peripheral neurotoxicity in patients with breast cancer: A randomized trial. J Natl Cancer Inst 112:55 62, 2020

39. Zhu Y, Yang J, Jiao S, et al: Ganglioside monosialic acid (GM1) prevents oxaliplatin induced peripheral neurotoxicity in patients with gastrointestinal tumors. World J Surg Oncol 11:19, 2013

40. Loprinzi CL, Hershman DL, Staff N: Neuronal protection for chemotherapy neuropathy prevention? J Natl Cancer Inst 112:3 4, 2020

41. Kuriyama A, Endo K: Goshajinkigan for prevention of chemotherapy induced peripheral neuropathy: A systematic review and meta analysis. Support Care Cancer 26:1051 1059, 2018

42. El Fatatry BM, Ibrahim OM, Hussien FZ, et al: Role of metformin in oxaliplatin induced peripheral neuropathy in patients with stage III colorectal cancer: Randomized, controlled study. Int J Colorectal Dis 33:1675 1683, 2018

43. Shinde SS, Seisler D, Soori G, et al: Can pregabalin prevent paclitaxel associated neuropathy? An ACCRU pilot trial. Support Care 24:547 553, 2016

44. de Andrade DC, Jacobsen Teixeira M, Galhardoni R, et al: Pregabalin for the prevention of oxaliplatin induced painful neuropathy: A randomized, double blind trial. Oncologist 22:1154 e105, 2017

45. Aghili M, Zare M, Mousavi N, et al: Efficacy of gabapentin for the prevention of paclitaxel induced peripheral neuropathy: A randomized placebo controlled clinical trial. Breast J 25:226 231, 2019

46. Durand JP, Deplanque G, Montheil V, et al: Efficacy of venlafaxine for the prevention and relief of oxaliplatin induced acute neurotoxicity: Results of EFFOX, a randomized, double blind, placebo controlled phase III trial. Ann Oncol 23:200 205, 2012

47. Zimmerman C, Atherton PJ, Pachman D, et al: MC11C4: A pilot randomized, placebo controlled, double blind study of venlafaxine to prevent oxaliplatin induced neuropathy. Support Care Cancer 24:1071 1078, 2016

48. Farshchian N, Alavi A, Heydarheydari S, et al: Comparative study of the effects of venlafaxine and duloxetine on chemotherapy induced peripheral neuropathy. Cancer Chemother Pharmacol 82:787 793, 2018

49. Schloss JM, Colosimo M, Airey C, et al: A randomised, placebo controlled trial assessing the efficacy of an oral B group vitamin in preventing the devel opment of chemotherapy induced peripheral neuropathy (CIPN). Support Care Cancer 25:195 204, 2017

50. Huang $\mathrm{H}, \mathrm{He} \mathrm{M}$, Liu L, et al: Vitamin $\mathrm{E}$ does not decrease the incidence of chemotherapy induced peripheral neuropathy: a meta analysis. Contemp Oncol (Pozn) 20:237 241, 2016

51. Salehi Z, Roayaei M: Effect of vitamin E on oxaliplatin induced peripheral neuropathy prevention: A randomized controlled trial. Int J Prev Med 6:104, 2015

52. Dhawan S, Andrews R, Kumar L, et al: A randomized controlled trial to assess the effectiveness of muscle strengthening and balancing exercises on chemotherapy induced peripheral neuropathic pain and quality of life among cancer patients. Cancer Nurs 43:269 280, 2020 
53. Molassiotis A, Suen LKP, Cheng HL, et al: A randomized assessor blinded wait list controlled trial to assess the effectiveness of acupuncture in the management of chemotherapy induced peripheral neuropathy. Integr Cancer Ther 18:1534735419836501, 2019

54. Lu W, Giobbie Hurder A, Freedman RA, et al: Acupuncture for chemotherapy induced peripheral neuropathy in breast cancer survivors: A randomized controlled pilot trial. Oncologist 25:310 318, 2020

55. D'Alessandro EG, Nebuloni Nagy DR, de Brito CMM, et al: Acupuncture for chemotherapy induced peripheral neuropathy: A randomised controlled pilot study. BMJ Support Palliat Care 10.1136/bmjspcare 2018001542 [epub ahead of print on June 29, 2019]

56. Rostock M, Jaroslawski K, Guethlin C, et al: Chemotherapy induced peripheral neuropathy in cancer patients: a four arm randomized trial on the effectiveness of electroacupuncture. Evid Based Complement Alternat Med 2013:349653, 2013

57. Han X, Wang L, Shi H, et al: Acupuncture combined with methylcobalamin for the treatment of chemotherapy induced peripheral neuropathy in patients with multiple myeloma. BMC Cancer 17:40, 2017

58. Hirayama $\mathrm{Y}$, Ishitani $\mathrm{K}$, Sato $\mathrm{Y}$, et al: Effect of duloxetine in Japanese patients with chemotherapy induced peripheral neuropathy: a pilot randomized trial. Int J Clin Oncol 20:866 871, 2015

59. Salehifar E, Janbabaei G, Hendouei N, et al: Comparison of the efficacy and safety of pregabalin and duloxetine in taxane induced sensory neuropathy: A randomized controlled trial. Clin Drug Investig 40:249 257, 2020

60. Smith TJ, Razzak AR, Blackford AL, et al: A pilot randomized sham controlled trial of MC5 A Scrambler Therapy in the treatment of chronic chemotherapy induced peripheral neuropathy (CIPN). J Palliat Care 35:53 58, 2020

61. Loprinzi C, Le Rademacher JG, Majithia N, et al: Scrambler therapy for chemotherapy neuropathy: A randomized phase II pilot trial. Support Care Cancer 28: 1183 1197, 2020

62. Gewandter JS, Chaudari J, Ibegbu C, et al: Wireless transcutaneous electrical nerve stimulation device for chemotherapy induced peripheral neuropathy: An open label feasibility study. Support Care Cancer 27:1765 1774, 2019

63. Lynch ME, Cesar Rittenberg P, Hohmann AG: A double blind, placebo controlled, crossover pilot trial with extension using an oral mucosal cannabinoid extract for treatment of chemotherapy induced neuropathic pain. J Pain Symptom Manage 47:166 173, 2014

64. Gewandter JS, Mohile SG, Heckler CE, et al: A phase III randomized, placebo controlled study of topical amitriptyline and ketamine for chemotherapy induced peripheral neuropathy (CIPN): A University of Rochester CCOP study of 462 cancer survivors. Support Care Cancer 22:1807 1814, 2014

65. Smith EM, Pang H, Cirrincione C, et al: Effect of duloxetine on pain, function, and quality of life among patients with chemotherapy induced painful peripheral neuropathy: A randomized clinical trial. JAMA 309:1359 1367, 2013

66. Rao RD, Michalak JC, Sloan JA, et al: Efficacy of gabapentin in the management of chemotherapy induced peripheral neuropathy: A phase 3 randomized, double blind, placebo controlled, crossover trial (NOOC3). Cancer 110:2110 2118, 2007

67. Mariani G, Garrone O, Granetto C, et al: Oxaliplatin induced neuropathy: could gabapentin be the answer? J Clin Oncol 19:609a, 2000 (abstr 2397)

68. Mitchell PL, Goldstein D, Michael M, et al: Addition of gabapentin to a modified FOLFOX regimen does not reduce oxaliplatin induced neurotoxicity. Clin Colorectal Cancer 6:146 151, 2006

69. Vondracek P, Oslejskova H, Kepak T, et al: Efficacy of pregabalin in neuropathic pain in paediatric oncological patients. Eur J Paediatr Neurol 13:332 336, 2009

70. Saif MW, Syrigos K, Kaley K, et al: Role of pregabalin in treatment of oxaliplatin induced sensory neuropathy. Anticancer Res 30:2927 2933, 2010

71. Nakashima T, Kiba T, Ogawa Y, et al: A case of paclitaxel induced peripheral neuropathy successfully treated with pregabalin [in Japanese]. Gan To Kagaku Ryoho 39:1443 1445, 2012

72. Nakashima T, Kiba T, Ogawa Y, et al: A case of neurotoxicity reduced with pregabalin in R CHOP chemotherapy for diffuse large B cell lymphoma [in Japanese]. Gan To Kagaku Ryoho 39:809 812, 2012

73. Nihei S, Sato J, Kashiwaba M, et al: Efficacy and safety of pregabalin for oxaliplatin and paclitaxel induced peripheral neuropathy [in Japanese]. Gan To Kagaku Ryoho 40:1189 1193, 2013

74. Nihei S, Sato J, Kimura T, et al: Treatment algorithm for oxaliplatin induced peripheral neuropathy [in Japanese]. Gan To Kagaku Ryoho 41:1387 1390, 2014

75. Lin NU, Bichkoff H, Hassett MJ: Increasing burden of prior authorizations in the delivery of oncology care in the United States. J Oncol Pract 14:525 528, 2018

76. Gewandter JS, Kleckner AS, Marshall JH, et al: Chemotherapy induced peripheral neuropathy (CIPN) and its treatment: An NIH Collaboratory study of claims data. Support Care Cancer 28:2553 2562, 2020

77. Monfort SM, Pan X, Patrick R, et al: Gait, balance, and patient reported outcomes during taxane based chemotherapy in early stage breast cancer patients. Breast Cancer Res Treat 164:69 77, 2017

78. Monfort SM, Pan X, Loprinzi CL, et al: Impaired postural control and altered sensory organization during quiet stance following neurotoxic chemotherapy: A preliminary study. Integr Cancer Ther 18:1534735419828823, 2019

79. Hu S, Huang KM, Adams EJ, et al: Recent developments of novel pharmacologic therapeutics for prevention of chemotherapy induced peripheral neuropathy. Clin Cancer Res 25:6295 6301, 2019

80. Janes K, Little JW, Li C, et al: The development and maintenance of paclitaxel induced neuropathic pain require activation of the sphingosine 1 phosphate receptor subtype 1. J Biol Chem 289:21082 21097, 2014

81. Stockstill K, Doyle TM, Yan X, et al: Dysregulation of sphingolipid metabolism contributes to bortezomib induced neuropathic pain. J Exp Med 215:1301 1313, 2018

82. Anand P, Elsafa E, Privitera R, et al: Rational treatment of chemotherapy induced peripheral neuropathy with capsaicin $8 \%$ patch: From pain relief towards disease modification. J Pain Res 12:2039 2052, 2019

83. Gilligan T, Coyle N, Frankel RM, et al: Patient clinician communication: American Society of Clinical Oncology consensus guideline. J Clin Oncol 35:3618 3632, 2017

84. Wang XS, Shi Q, Bhadkamkar NA, et al: Minocycline for symptom reduction during oxaliplatin based chemotherapy for colorectal cancer: A phase II randomized clinical trial. J Pain Symptom Manage 58:662 671, 2019

85. Pachman DR, Dockter T, Zekan PJ, et al: A pilot study of minocycline for the prevention of paclitaxel associated neuropathy: ACCRU study RU221408I. Support Care Cancer 25:3407 3416, 2017

86. Kneis S, Wehrle A, Müller J, et al: It's never too late balance and endurance training improves functional performance, quality of life, and alleviates neuropathic symptoms in cancer survivors suffering from chemotherapy induced peripheral neuropathy: Results of a randomized controlled trial. BMC Cancer 19:414, 2019 


\section{AUTHORS' DISCLOSURES OF POTENTIAL CONFLICTS OF INTEREST}

\section{Prevention and Management of Chemotherapy-Induced Peripheral Neuropathy in Survivors of Adult Cancers: ASCO Guideline Update}

The following represents disclosure information provided by authors of this manuscript. All relationships are considered compensated unless otherwise noted. Relationships are self held unless noted. I = Immediate Family Member, Inst = My Institution. Relationships may not relate to the subject matter of this manuscript. For more information about ASCO's conflict of interest policy, please refer to www.asco.org/rwc or ascopubs.org/jco/authors/author center.

Open Payments is a public database containing information reported by companies about payments made to US licensed physicians (Open Payments).

\section{Charles L. Loprinzi}

Consulting or Advisory Role: PledPharma, Metys Pharmaceuticals, Asahi Kasei, Disarm Therapeutics, OnQuality Pharmaceuticals, NKMax, Mitsubishi Tanabe Pharma

Research Funding: Bristol Myers Squibb (Inst)

Other Relationship: Hologic/Cynosure

Jonathan Bleeker

Consulting or Advisory Role: Bristol Myers Squibb (Inst)

Travel, Accommodations, Expenses: Bristol Myers Squibb, PledPharma

Guido Cavaletti

Consulting or Advisory Role: PledPharma, Seattle Genetics, Toray Industries, Sonnet BioTherapeutics, Disarm Therapeutics, Helsinn Healthcare

Daniel L. Hertz

Research Funding: Disarm Therapeutics

Uncompensated Relationships: PEPID, Saladax Biomedical

Mark R. Kelley

Leadership: Apexian Pharmaceuticals

Stock and Other Ownership Interests: Apexian Pharmaceuticals

Consulting or Advisory Role: Apexian Pharmaceuticals, Ocuphire Pharma

Patents, Royalties, Other Intellectual Property: I have a number of antibodies that have been licensed from Indiana University School of Medicine that are sold by various companies. The royalties come back to the school and I share in some of them. I receive some royalties from licensed technology to Apexian

Pharmaceuticals and could eventually receive royalties from Ocuphire Pharma if milestones are met. Ocuphire is an eye company and not cancer related. I have not received any royalties from these units at this time beyond consulting as disclosed. Apexian licensed my IP and then sublicensed IP to Ocuphire for the eye.
Maryam B. Lustberg

Consulting or Advisory Role: PledPharma, Disarm Therapeutics

Other Relationship: Hologic/Cynosure

Ellen M. Lavoie Smith

Consulting or Advisory Role: Mundipharma Research, Disarm Therapeutics Research Funding: National Institutes of Health/National Cancer Institute (Inst), Disarm Therapeutics (Inst)

Mary Lou Smith

Consulting or Advisory Role: Novartis, Pfizer

Research Funding: Genentech (Inst), Celgene (Inst), Genomic Health (Inst), Novartis (Inst), Foundation Medicine (Inst)

Travel, Accommodations, Expenses: Genentech/Roche

Thomas J. Smith

Employment: UpToDate

Patents, Royalties, Other Intellectual Property: Royalties from Oxford Textbook of Cancer Communication, co editor.

Open Payments Link: https://openpaymentsdata.cms.gov/physician/202382/ general payments

Nina Wagner-Johnston

Consulting or Advisory Role: ADC Therapeutics, Bayer, Regeneron, CALIB R, Verastem, Gilead Sciences

Research Funding: Merck (Inst), Novartis/Pfizer (Inst), Genentech (Inst), Astex Pharmaceuticals (Inst), Juno Therapeutics (Inst), Regeneron (Inst), Acerta (Inst), ADC Therapeutic (Inst)

\section{Dawn L. Hershman}

Consulting or Advisory Role: AIM Specialty Health

No other potential conflicts of interest were reported. 
TABLE A1. Prevention and Management of Chemotherapy Induced Peripheral Neuropathy in Survivors of Adult Cancers Guideline Update Expert Panel Membership

Name

Affiliation/Institution

Role/Area of Expertise

\begin{tabular}{|c|c|c|}
\hline Charles L. Loprinzi, MD (co chair) & Mayo Clinic, Rochester, MN & Medical oncology \\
\hline Dawn L. Hershman, MD, MS (co chair) & Columbia University Medical Center, New York, NY & Medical oncology \\
\hline Maryam B. Lustberg, MD & Ohio State University, Columbus, $\mathrm{OH}$ & Medical oncology \\
\hline Nina Wagner Johnston, MD & Johns Hopkins, Baltimore, MD & Medical oncology \\
\hline Bryan P. Schneider, MD & Indiana University School of Medicine, Indianapolis, IN & Medical oncology \\
\hline Shelby Terstriep, MD & Sanford Health, Fargo, ND & Medical oncology \\
\hline Judith A. Paice, PhD, RN & Northwestern University, Chicago, IL & Nursing/pain \\
\hline Guido Cavaletti, MD, PhD & University of Milano Bicocca, Monza, Italy & Neurology/pain \\
\hline Daniel L. Hertz, PharmD, PhD & University of Michigan, Ann Arbor, MI & Clinical pharmacology \\
\hline $\begin{array}{l}\text { Cynthia Chauhan, MSW, Patient } \\
\text { Advocate }\end{array}$ & Wichita, KS & Patient representative \\
\hline $\begin{array}{l}\text { Mary Lou Smith, JD, MBA, Patient } \\
\text { Advocate }\end{array}$ & Research Advocacy Network, Plano, TX & Patient representative \\
\hline Christina Lacchetti, MHSc & ASCO, Alexandria, VA & $\begin{array}{l}\text { ASCO Practice Guideline Staff (Health Research } \\
\text { Methods) }\end{array}$ \\
\hline
\end{tabular}

Abbreviation: PGIN, Practice Guideline Implementation Network. 